\title{
What is the impact on health and wellbeing of interventions that foster respect and social inclusion in community- residing older adults? A systematic review of quantitative and qualitative studies
}

\author{
S. Ronzi ${ }^{1 *}$ (D) L. Orton ${ }^{1}$, D. Pope ${ }^{1}$, N. K. Valtorta ${ }^{2}$ and N. G. Bruce
}

\begin{abstract}
Background: Many interventions have been developed to promote respect and social inclusion among older people, but the evidence on their impacts on health has not been synthesised. This systematic review aims to appraise the state of the evidence across the quantitative and qualitative literature.

Methods: Eligible studies published between 1990 and 2015 were identified by scanning seven bibliographic databases using a pre-piloted strategy, searching grey literature and contacting experts. Studies were included if they assessed the impact (quantitatively) and/or perceived impact (qualitatively) of an intervention promoting respect and social inclusion on the physical or mental health of community-residing people aged 60 years and older. Titles and abstracts were screened for eligibility by one reviewer. A second reviewer independently screened a $10 \%$ random sample. Full texts were screened for eligibility by one reviewer, with verification by another reviewer. Risk of bias was assessed using standardised tools. Findings were summarised using narrative synthesis, harvest plots and logic models to depict the potential pathways to health outcomes.
\end{abstract}

Results: Of the 27,354 records retrieved, 40 studies (23 quantitative, 6 qualitative, 11 mixed methods) were included. All studies were conducted in high and upper middle-income countries. Interventions involved mentoring, intergenerational and multi-activity programmes, dancing, music and singing, art and culture and information-communication technology. Most studies $(n=24)$ were at high or moderate risk of bias. Music and singing, intergenerational interventions, art and culture and multi-activity interventions were associated with an overall positive impact on health outcomes. This included depression ( $n=3)$, wellbeing $(n=3)$, subjective health $(n=2)$, quality of life $(n=2)$, perceived stress and mental health $(n=2)$ and physical health $(n=2)$. Qualitative studies offered explanations for mediating factors (e.g. improved self-esteem) that may lead to improved health outcomes and contributed to the assessment of causation.

Conclusions: Whilst this review suggests that some interventions may positively impact on the health outcomes of older people, and identified mediating factors to health outcomes, the evidence is based on studies with heterogeneous methodologies. Many of the interventions were delivered as projects to selected groups, raising important questions about the feasibility of wider implementation and the potential for population-wide benefits.

Systematic review registration: PROSPERO registration number CRD42014010107

Keywords: Social inclusion, Older people, Ageing, Systematic review, Health impact, Age-friendly environments

\footnotetext{
* Correspondence: ronzis@liverpool.ac.uk

'Department of Public Health and Policy, University of Liverpool, Liverpool, UK

Full list of author information is available at the end of the article
} 


\section{Background}

According to the World Population Ageing report [1], the world's population aged 60 years and older is expected to increase to more than two billion by 2050; by 2030, one in six people will be 60 years or older A growing number of these older people live in urban environments, with particularly rapid increases in low- and middle-income countries [2]. The combination of population ageing and urbanisation places increasingly complexes demands on health and social care systems, raising significant challenges for welfare systems worldwide [3, 4].

The older population should be a net asset to society, but suitable policies and services in place will be required to realise this [4-6]. In the Global Strategy and Action Plan on Ageing and Health, published in 2016, the WHO advocated the development of physical and social settings that support older people to live independently and in good health for longer but also optimise health and wellbeing for the wider community [7]. Age-friendly environments aim to facilitate older adults' access to opportunities for social interaction and engagement with cultural and social resources (e.g. libraries and green spaces) [8, 9].

A range of interventions have been developed to create age-friendly environments, based on eight different domains theorised by the WHO as having a potential impact on health and wellbeing [10]. One of these domains is respect and social inclusion, which has been considered of fundamental importance to older people in qualitative research [10-14] and in national and international policy $[4,6,7,15,16]$. Persistent disrespectful attitudes and misconceptions about older people and growing old are acknowledged as being a significant barrier to the development of good public health policies on ageing $[7,17]$. They lead to negative perceptions of ageing (e.g. by disregarding the contribution older people make to society) and can negatively impact health and wellbeing in later life [18-20]. For instance, Levy et al. [21] have shown that older people who were exposed to negative age stereotypes were less likely to recuperate from disability than those exposed to more positive self-perceptions of ageing. Moreover, people who internalised negative age stereotypes sooner in life were more likely to experience cardiovascular events in the coming 38 years than those who had more positive selfperceptions of ageing [22].

The term social inclusion has explicit links with concepts such as equality, human rights and social cohesion, and it has focused on barriers that prevent people from participating meaningfully in society [19]. By focusing on goals rather than problems, the concept of inclusion adopts a positive approach [23, 24]. It is not merely the implied opposite of social exclusion but refers to the opportunities for individuals to cultivate social relationships, have access to resources and feel part of the community they live in $[25,26,27]$. Respect in relation to older people, meanwhile, refers to positive attitudes and behaviours towards the elderly, so that they may feel accepted, valued and appreciated by the community regardless of age [28].

Whilst many interventions to promote respect and social inclusion in older people have been developed, the evidence on their impacts on health and wellbeing has not been synthesised. One of the reasons for this limited synthesis owes to complexity of these interventions [29]. In this context, complexity may arise by the various interactions between the components that may be involved in the intervention and its context, and external factors. For instance, an intervention may indirectly improve the level of social engagement of older adults and, in turn, their wellbeing and quality of life [2]. The same intervention may also consist of relatively well-defined initiatives (e.g. reading activities) or may be a much more complex set of actions driven by policy (e.g. different reading activities in various schools). These are some of the reasons that make the assessment and synthesis of these interventions particularly challenging [30-39]. The WHO has identified synthesising the evidence on interventions promoting age-friendly environments as a key priority to establish what is known about the impacts of these [7]. Responding to this call, this systematic review attempts to synthesise the evidence of health impacts of interventions on respect and social inclusion in older people. It addresses the following research question: What is the empirical evidence on the impact on health and wellbeing of interventions which foster respect and social inclusion in community-residing older adults? The aims were to (i) identify and understand the health impacts of interventions that aim to promote respect and social inclusion among older people and (ii) to elucidate the complex pathways that may lead to improved health outcomes.

\section{Methods}

We followed the Centre for Reviews and Dissemination's guidance for undertaking reviews in healthcare [40]. The Preferred Reporting Items for Systematic Reviews and Meta-Analyses (PRISMA) guidelines informed our reporting [41]. The protocol was registered with the PROSPERO database [42], and a PRISMA checklist is available as Additional file 1.

The first step we took, before searching the literature, was to develop logic models depicting the possible multiple interacting pathways through which the interventions could affect health and wellbeing [35, 43-45], as recommended in the literature on evidence synthesis of complex interventions [36, 43, 46-50]. First, we conducted scoping work (which involved looking at existing literature reviews $[51,52]$ and key background literature $[10,14,53,54]$ on respect and social inclusion and age- 
friendly environments) to identify interventions, outcomes and mediating factors that were mentioned in relation to promoting respect and social inclusion in older people.

Two main types of interventions emerged: (1) intergenerational interventions and (2) information and communication technology interventions. For these two intervention types, we developed logic models at the start of the review process, based on the pathways mentioned in the literature; we then went on to adapt them over the course of the review process, to incorporate the additional information we identified. Please refer to Figs. 3 and 4, in the "Results", for an example of the logic models for intergenerational interventions.

For interventions which were not identified from our scoping review, but which met our inclusion criteria (e.g. they were qualified as interventions promoting respect and social inclusion, such as music and singing activities), we generated logic models after studies were assessed for inclusion. These models were based on the information reported in the included studies about mediating factors and pathways. For further details on the synthesis process, please refer to the "Synthesis" section.

\section{Search strategy}

We developed and piloted a search strategy designed to capture the most relevant evidence to address the research question. We searched eight electronic bibliographic databases: Scopus, MEDLINE, PsycINFO, CINAHL and Web of Science Core Collection: citation indexes (Social Sciences Citation Index, Science Citation Index, Book Citation Index-Science, Book Citation Index-Social Sciences and Humanities); the Web of Science Core Collection: citation indexes (Conference Proceedings Citation Index-Science, Conference Proceedings Citation Index-Social Science and Humanities); the Cochrane Library: Cochrane Reviews (Reviews and Protocols) and other reviews and trials (ProQuest Dissertations \& Thesis). Searches comprised a combination of subject terms selected from the controlled vocabulary or thesaurus where possible (MEDLINE, CINAHL and PsycINFO) and a wide range of free-text terms. For the full electronic strategy used to search MEDLINE, see Additional file 2. Relevant systematic reviews were retrieved, and titles of individual studies were checked to see if they met the inclusion criteria.

We searched sources of grey literature including policy papers and reports from the following: the Joseph Rowntree Foundation (http://www.jrf.org.uk/), Age UK (http:// www.ageuk.org.uk/), Age of Creativity (http://www.ageof creativity.co.uk/), Alzheimer's Association (http://www.alz heimers.org.uk/), InterGen (http://fromgeneration2genera tion.org.uk/), Beth Johnson Foundation (http://www.bjf. org.uk/) and Manchester Institute for Collaborative Research on Ageing (http://www.micra.manchester.ac.uk). We checked the list of references of potentially relevant papers included as full text if the title met the inclusion criteria. We also contacted topic experts to identify any additional data sources.

Searches were restricted to the English language as there were no resources for translation. We were interested in the literature relevant to contemporary social and political contexts of ageing and respect and social inclusion. The aim of our review was to identify evidence about interventions which could be implemented in the context of current efforts to promote age-friendly environments. We therefore chose the 1990 as the start date of our searches (up until January 2015, when the search was conducted) to coincide with the emergence of debates about, and initiatives aimed at, designing optimum community environments for ageing populations [55].

\section{Inclusion criteria}

1. Population: Studies where at least $50 \%$ of participants were aged $60+$ years were eligible for the review. Those where some of the population were younger than 60 years were included if the data for subgroups of older people (60+ years) could be disaggregated or where the average age was over 60 .

2. Interventions: Any intervention aiming to improve respect and social inclusion in older people was included. Studies were included if they did not explicitly mention either term but where the purpose of the intervention was to improve community inclusion, social participation, sense of belonging, access to learning, cultural and social opportunities or social relationships in the community. We only included mentoring interventions where the aim was to engage older people in social activities with others within a group setting. By contrast, befriending interventions focus on improving the level of social support and decreasing loneliness through one-to-one interaction [56]. Because the latter is not a group- or communitybased activity, it did not meet our inclusion criteria.

3. Control groups: Relevant comparison groups included (i) older people not exposed to the intervention being investigated, (ii) older people exposed to other forms of interventions included as usual practice and (iii) older people exposed to other interventions for respect and social inclusion. This criterion applied only to quantitative studies.

4. Outcomes assessed quantitatively: Health outcomes pertinent to the review included any measure of physical and mental health of participants, healthrelated quality of life and measures of wellbeing. Standardised outcome measures were defined as those supported by an academic reference and evidence of their psychometric properties. Non- 
standardised health outcome measures were defined as those developed by the authors for the purposes of the study. Although we recognised that cognitive function is a health outcome, through our logic models, outcomes related to cognitive function (e.g. memory and language attention) were included only if there was evidence that the intervention (e.g. Internet training) increased respect and social inclusion and that this led to the improved outcome. Likewise, outcomes related to autonomy and physical activity (e.g. posture, balance, muscle strength, stability and walking speed) were included only if there was evidence that the intervention (e.g. dancing classes) increased respect and social inclusion and that this led to the improved outcome.

5. Setting: Only studies conducted in community settings were included in the review. Studies that included groups from both community and institutionalised settings (e.g. nursing homes) were included if the community data could be disaggregated.

6. Study design: All empirical study designs including quantitative designs (randomised and non-randomised controlled studies, before and after studies), mixed methods design and qualitative designs were eligible for the review. Case studies (defined as "drawing on multiple sources of information to provide a broad evaluation of a specific project, program, or policy" ([52] p. 122) were only included if sampling techniques, data collection methods and results/ analysis of health impacts could be ascertained.

\section{Screening and selection}

Search results were downloaded into EPPI-Reviewer 4 software [57]. After removing duplicates, titles and abstracts were screened for eligibility by one reviewer (SR), using a pre-designed and piloted tool based on the inclusion criteria. A second reviewer (NKV) independently screened a $10 \%$ random sample of titles and abstracts. The level of agreement was checked using EPPI-Reviewer 4 software. This produced a reconciliation report showing that there was less than $2 \%$ disagreement out of 2736 papers independently coded by the two reviewers (SR and NKV). Disagreements were resolved through discussion or by recourse to a third reviewer (LO/DP/NB). One reviewer (SR) screened full-text papers for eligibility with $15 \%$ screened by another reviewer (LO/DP/NB) where there was uncertainty about the relevance for inclusion. Any discrepancies were resolved through discussion, and decisions for exclusion were documented.

\section{Data extraction and risk of bias assessment}

A single reviewer (SR) conducted data extraction for included studies using separate pre-piloted forms for quantitative and qualitative evidence; one reviewer (DP/ LO/NB) checked $15 \%$ of data extraction tables. Extracted information included (i) bibliographic details, (ii) study design, (iii) study participants including details of control groups for quantitative studies, (iv) aims and key features of the intervention, (v) outcomes and outcome measures, (vi) main results, (vii) main conclusions and (viii) key methodological issues. From the qualitative studies, we extracted participants' own narratives and then summarised these data in a concise message in data extraction tables. The summary included information on factors (e.g. improved self-esteem) reported by older people on the impact of the intervention on their health and wellbeing.

All studies were critically appraised by one reviewer (SR). We assessed risk of bias (RoB) and methodological quality using different methods for quantitative and qualitative studies, as explained below. For shorthand, we reported the overall assessment of quality as RoB throughout this paper and we used it as preferred terminology [52]. In the summary tables (Additional files 4 and 5), we used a global assessment for quantitative and qualitative studies. This was used to facilitate reporting of the data in the summary tables and give an indication of the RoB among the different studies. As recommended by the literature [58], we incorporated the RoB assessments into the findings (please refer to the "Results" section). For the item-level RoB assessment for each study, please refer to Additional file 6 (quantitative studies) and Additional file 7 (qualitative studies). Case studies were assessed using an adapted version of Atkins \& Sampson's tool [59]. Quantitative studies and quantitative elements of mixed method studies were assessed using the Liverpool Quality Assessment Tools (LQATs) [60]. The forms include (i) selection procedures, (ii) baseline assessment, (iii) outcome assessment, (iv) analysis/confounding and (v) contribution of evidence towards the review question that are rated as strong, moderate or weak. Qualitative studies and qualitative elements of mixed methods studies were appraised using an adapted version of Harden et al. [61, 62] and Mays and Pope [63] tools. The form is divided into sections covering study context, methodology, use of strategies to increase reliability and validity and extent to which findings reflected participant perspectives and experiences. A global assessment of validity was made based on whether aspects of the study were clear, adequate or explicit using this scale.

\section{Synthesis}

The broad focus of interventions fostering respect and social inclusion, and the heterogeneity across study designs and outcomes, precluded meta-analysis [42]. We therefore conducted a narrative synthesis $[40,64]$ comprising four elements: 
1. We grouped and tabulated studies according to the type of intervention evaluated. A broad range of interventions were identified, including those based on (i) mentoring, (ii) intergenerational programmes, (iii) dancing, (iv) music and singing, (v) art and culture, (vi) information-communication technology and (vii) multi-activity programmes (e.g. health promotion). To facilitate reporting of the data in the summary tables (Additional files 4 and 5) and to give an indication of the potential RoB among the different studies, we ranked quantitative and qualitative studies based on a global assessment (from lower to higher RoB).

2. For each intervention category, we produced a narrative summary of findings, grouping studies according to whether they produced similar results, measured the same outcomes and/or shared a theoretical basis [64]. RoB was discussed in each narrative summary [58].

3. We used harvest plots to graphically represent the quantitative findings, including RoB for each intervention (Table 1). These plots represent an overall summary of the quantity, direction and strength of the evidence for the various health outcomes [47].

4. Logic model development:

As explained earlier, based on scoping work, we generated logic models for (1) intergenerational interventions and 2) information and communication technology interventions. The initial construction of the logic models (pre-review) helped us to conceptualise possible outcomes and mechanisms through which interventions on social inclusion might produce effects on health outcomes. Successively, based on the evidence retrieved, we assessed whether the mediating factors and outcomes that we depicted in the initial logic models were supported by the evidence (see Figs. 3 and 4).

Diagram development:

Diagrams were developed during the narrative synthesis process. They represent a descriptive overview of the quantitative and qualitative evidence retrieved for each intervention type.

The mediating factors included in the diagrams came from the participants' own narratives that emerged from the qualitative studies (on how older people reported the impact of the intervention). They offer some explanations about possible mechanisms through which interventions on respect and social inclusion may impact on older people's health (e.g. feeling valued). The diagrams also present the list of outcomes being studied by the qualitative and quantitative studies (including number of studies), and the effect for quantitative studies (see Figs. 5, 6, 7, 8, 9 and 10). We have not included the assessed RoB in these diagrams, as the RoB is presented in the harvest plot (Table 1).

\section{Results \\ Study selection}

Of the initial 27,354 references retrieved, 259 were filtered for full-text paper review after screening titles and abstracts. Of these, 45 records based on 40 studies (23 quantitative, 6 qualitative, 11 mixed methods) met the inclusion criteria of

Table 1 Harvest plot for interventions on respect and social inclusion in older people

\begin{tabular}{|c|c|c|c|c|c|c|c|c|c|c|c|c|c|c|c|c|c|c|c|c|c|}
\hline & \multicolumn{3}{|c|}{ Mentoring } & \multicolumn{3}{|c|}{ Intergenerational } & \multicolumn{3}{|c|}{ Dancing } & \multicolumn{3}{|c|}{ Music and singing } & \multicolumn{3}{|c|}{$\begin{array}{c}\text { Information- } \\
\text { communication } \\
\text { technology }\end{array}$} & \multicolumn{3}{|c|}{ Art and Culture } & \multicolumn{3}{|c|}{ Multi-activity } \\
\hline & $\begin{array}{l}\text { Favours } \\
\text { contr. }\end{array}$ & $\begin{array}{l}\text { No } \\
\text { effect }\end{array}$ & $\begin{array}{l}\text { Favours } \\
\text { int. }\end{array}$ & $\begin{array}{l}\text { Favours } \\
\text { contr. }\end{array}$ & $\begin{array}{l}\text { No } \\
\text { effect }\end{array}$ & $\begin{array}{l}\text { Favours } \\
\text { int. }\end{array}$ & $\begin{array}{l}\text { Favours } \\
\text { contr. }\end{array}$ & $\begin{array}{l}\text { No } \\
\text { effect }\end{array}$ & $\begin{array}{l}\text { Favours } \\
\text { int. }\end{array}$ & $\begin{array}{l}\text { Favours } \\
\text { contr. }\end{array}$ & $\begin{array}{l}\text { No } \\
\text { effect }\end{array}$ & $\begin{array}{l}\text { Favours } \\
\text { int. }\end{array}$ & $\begin{array}{l}\text { Favours } \\
\text { contr. }\end{array}$ & $\begin{array}{l}\text { No } \\
\text { effect }\end{array}$ & $\begin{array}{l}\text { Favours } \\
\text { int. }\end{array}$ & $\begin{array}{l}\text { Favours } \\
\text { contr. }\end{array}$ & $\begin{array}{l}\text { No } \\
\text { effect }\end{array}$ & $\begin{array}{l}\text { Favours } \\
\text { int. }\end{array}$ & $\begin{array}{l}\text { Favours } \\
\text { contr. }\end{array}$ & $\begin{array}{l}\begin{array}{l}\text { No } \\
\text { effect }\end{array} \\
\end{array}$ & $\begin{array}{l}\text { Favours } \\
\text { int. }\end{array}$ \\
\hline Depression & & 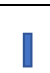 & & & a & Ш & & घ & & & B & I & & b & & & Ш & & & & प \\
\hline Anxiety & & & & & & & & & & & & 7 & & $\pi$ & & & & & & & \\
\hline $\begin{array}{l}\text { Perceived } \\
\text { stress }\end{array}$ & & & & & & & & & & & & 7 & & & & & & & & & 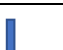 \\
\hline $\begin{array}{l}\text { Mental } \\
\text { health }\end{array}$ & & $b$ & & & घ & & & & & & & 口 & & 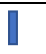 & & & ] & & & & ] \\
\hline $\begin{array}{l}\text { Subjective } \\
\text { health }\end{array}$ & $\Pi$ & & & & $\prod$ & 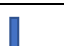 & & 口 & & & & & & & & & & m & & & h \\
\hline $\begin{array}{l}\text { Physical } \\
\text { health }\end{array}$ & & $b$ & & & घ & & & & & & 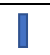 & 口 & & 1 & & & & ] & & & \\
\hline Wellbeing & & & & & & & & घ & & & $\pi$ & ם & & & & & 口 & ] & & $B$ & 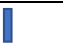 \\
\hline $\begin{array}{l}\text { Quality of } \\
\text { life }\end{array}$ & & $\square$ & & & घ & ш & & & & & 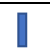 & घ & & 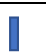 & घ & & 】 & & & & \\
\hline Falls & & & & & घ & & & घ & घ & & & $\pi$ & & & & & & $\bar{T}$ & & & \\
\hline $\begin{array}{l}\text { Chronic } \\
\text { pain }\end{array}$ & & & & & & & & & & & & & & & & & & 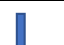 & & & \\
\hline
\end{tabular}

Each solid bar represents a study. The height of the bar reflects the RoB assigned to that study (high bar, low/low-moderate RoB; medium bar, moderate RoB; low bar, moderate-high/high RoB), so that the strength of the evidence could be determined, and greater weight is given to conclusions from the most methodological robust and reliable studies. See "Methods" for assessing RoB in quantitative studies 
the review (Fig. 1). The PRISMA flow diagram of the study selection process is shown in Fig. 1.

\section{Study characteristics}

Additional file 4 summarises the characteristics of the quantitative studies, and Additional file 5 presents the characteristics of the qualitative studies. Table 1 shows the harvest plot, which represents a brief overview of the strength of the quantitative evidence for the various health outcomes and the RoB of the studies. In Fig. 2, the number of qualitative and quantitative studies is stratified by intervention category $(n=40)$.

Studies using mixed methods designs contributed to both quantitative and qualitative evidence. Thirty-four studies provided quantitative evidence and 14 studies qualitative evidence. One study [65] contributed to both the mentoring and intergenerational interventions, and another [66] contributed to both singing and art and culture interventions.

\section{Study design}

Of the 34 studies reporting quantitative evidence, seven adopted individual or cluster randomised controlled trials (RCTs), with the rest using quasi-experimental designs-four were controlled before and after studies, seven were cluster or individual controlled studies and 15 were uncontrolled before and after studies. Studies reported a range of comparison/control groups including other interventions not related to respect and social inclusion (e.g. weekly recreational activities) $(n=2)$, usual care (e.g. through routinely available health, social and voluntary care services) $(n=2)$, other activities (e.g. hobbies) $(n=5)$ and older people selected from waiting lists $(n=2)$. One study used multiple comparison groups [67].

Most studies had only one follow-up assessment conducted between 2 weeks and 8 months after initiation of the intervention/baseline measurements.

Of the 14 studies reporting qualitative evidence, the methods used included the following: focus group discussions $(n=3)$, interviews $(n=7)$, a mix of focus groups

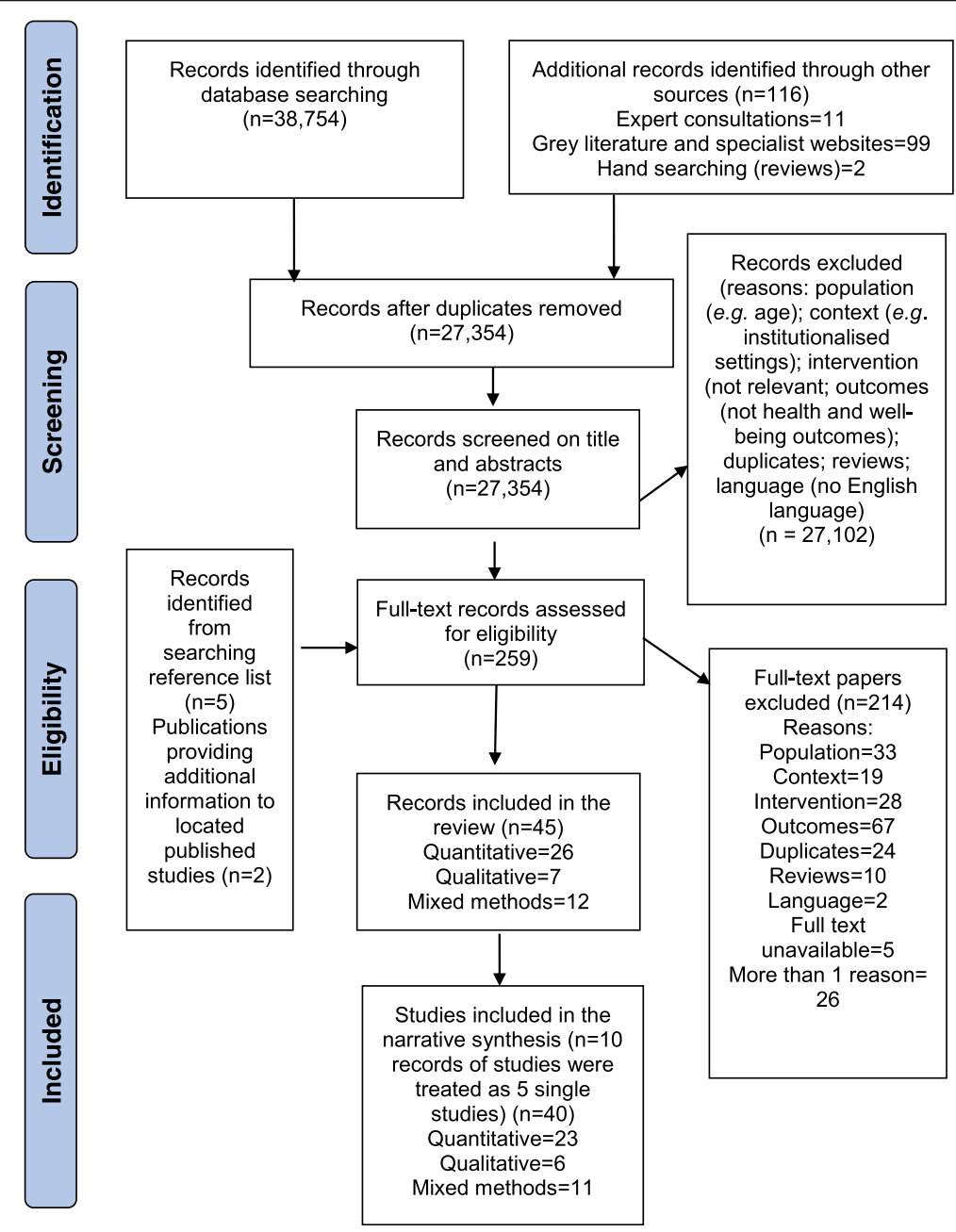

Fig. 1 PRISMA flow diagram of the study selection process 


\section{Quantitative and qualitative studies stratified by intervention category}

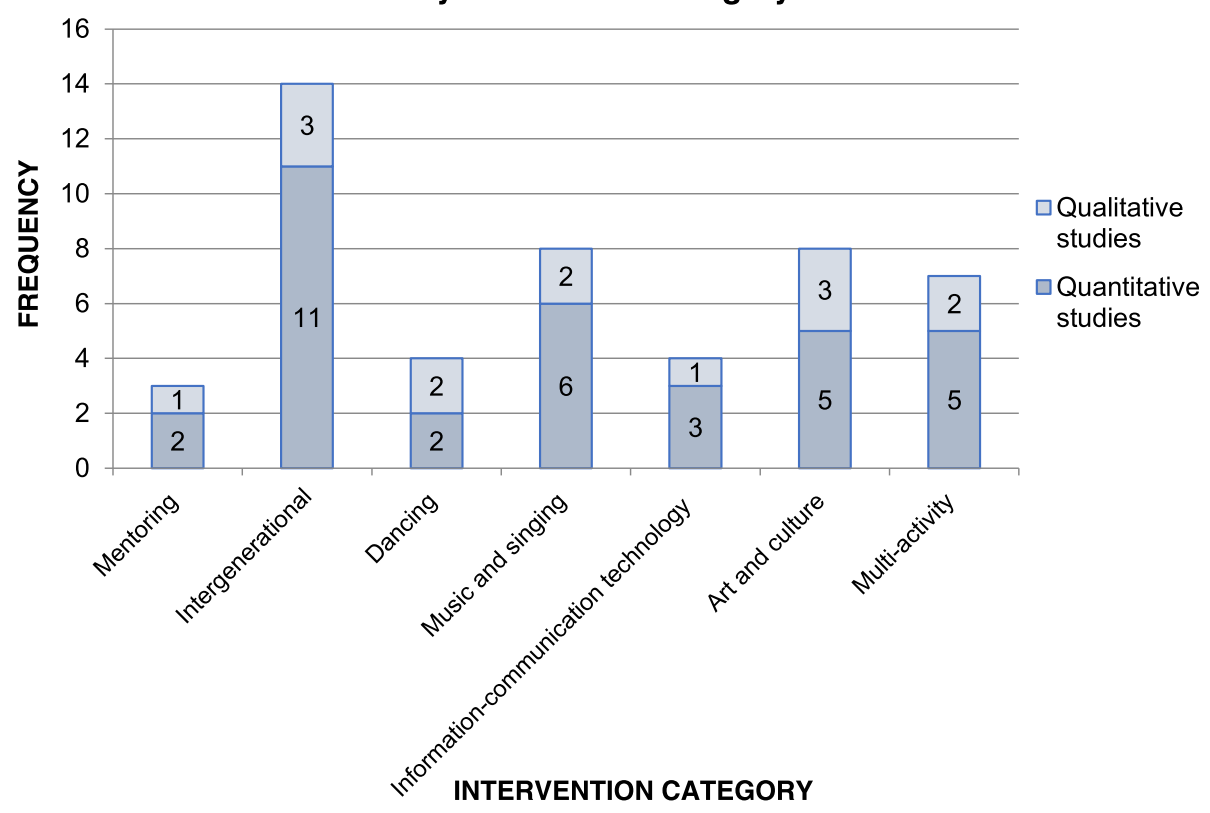

Fig. 2 Quantitative and qualitative studies stratified by intervention category

and interviews $(n=2)$, diary writing $(n=1)$, observation $(n=1)$ and qualitative comments offered in response to open questions included in the questionnaires $(n=1)$.

\section{Setting}

All studies concerned higher and upper middle-income countries. Thirteen were from the UK; 13 from the USA; three from Japan; two each from the Netherlands, Australia, Canada and Brazil; and one each from Spain, Italy and China.

\section{Population}

The majority of studies included healthy older people aged between 60 and 95 years, with the exception of two studies that included older people with dementia $[68,69]$ and three studies that included older people with Parkinson's disease [70-72].

Most studies comprised a majority of women, with only one study reporting an even balance between women and men [65] and one study including women only [73]. In most studies, participants were either volunteers currently involved with/interested in the programme or those recruited through fliers and letters. Study participants were also referred by general practices [74] or recruited from day centres [68] and community centres/groups [75, 76].

\section{Delivery and frequency of contacts}

Four studies included interventions delivered by peers [7780], eight were led by the study participants themselves [65,
$76,81-86]$, one involved both professionals and students [87], four were led by study participants with some support from help desk and community centres [67, 88-90] and 19 studies were led by professionals $[66,69-75,91-103]$.

The frequency of contact with participants varied, with most interventions being delivered on a weekly or other periodic basis (e.g. every 2 weeks). Most interventions lasted between 3 and 12 weeks, with a few lasting for extended periods (26 weeks [73], 30 weeks [66] and 3 years [96]). In one study, the intervention duration was not clear [85].

\section{Outcomes}

Impacts were reported on the following: depression $(n=20)$, subjective health $(n=7)$, mental health $(n=4)$, wellbeing $(n=8)$, physical health $(n=7)$, quality of life $(n=7)$, falls $(n=4)$, perceived stress and anxiety $(n=3)$ and chronic pain $(n=1)$. See Additional file 3 for an overview of the scales used for the quantitative studies in measuring outcomes. Most of the included studies used standardised scales, with only a few studies using non-standardised measures for subjective health $[66,79,82,83]$, falls $[70,76]$ and quality of life outcomes $[78,95]$.

\section{RoB}

Overall, 12 studies were rated as high and medium-high RoB [65, 69, 70, 72-74, 76, 78, 85, 95, 103, 104], 12 studies as moderate RoB [70, 71, 75, 80, 83, 84, 93, 94, 97, 98, 100, $102]$ and 21 as low or low-moderate RoB [66-68, 77, 79, 81, $82,86,87,89,90,92,96,98-100,105-107]$. The main RoB issues with these studies included small sample size, poor 
selection of participants and differences observed between intervention and control group participants at baseline.

\section{Mediating factors}

Of the 14 studies reporting qualitative evidence, the most common mediating factors explored were the following: improved self-confidence and self-esteem, feeling valued, improved social relationships and interactions, reduction of social isolation, feeling of happiness and enjoyment and feeling more physically active.

\section{Development of logic models: pre and post review}

To illustrate how the logic models evolved thorough the review process, Fig. 3 shows the logic model that we initially developed for intergenerational interventions (pre review).

Based on the evidence retrieved, we assessed whether the hypothesised mediating factors and outcomes were supported by the evidence. As shown in the final version of the logic model (Fig. 4), through the review, we were able to identify some of the activities that constitute intergenerational interventions (e.g. reading books to children and assisting young people in school activities). From the quantitative evidence, which looked at the impact of the interventions, we generated some additional outcomes (e.g. depression). From the qualitative data, which provided information on how interventions might work according to older people's narratives, we generated some additional mediating factors (e.g. feeling valued) that could be involved in the process of improving health outcomes.

\section{Results by intervention category}

For each intervention category, the number of studies, the type of study design and the RoB for each study are presented with a summary of the main findings (both

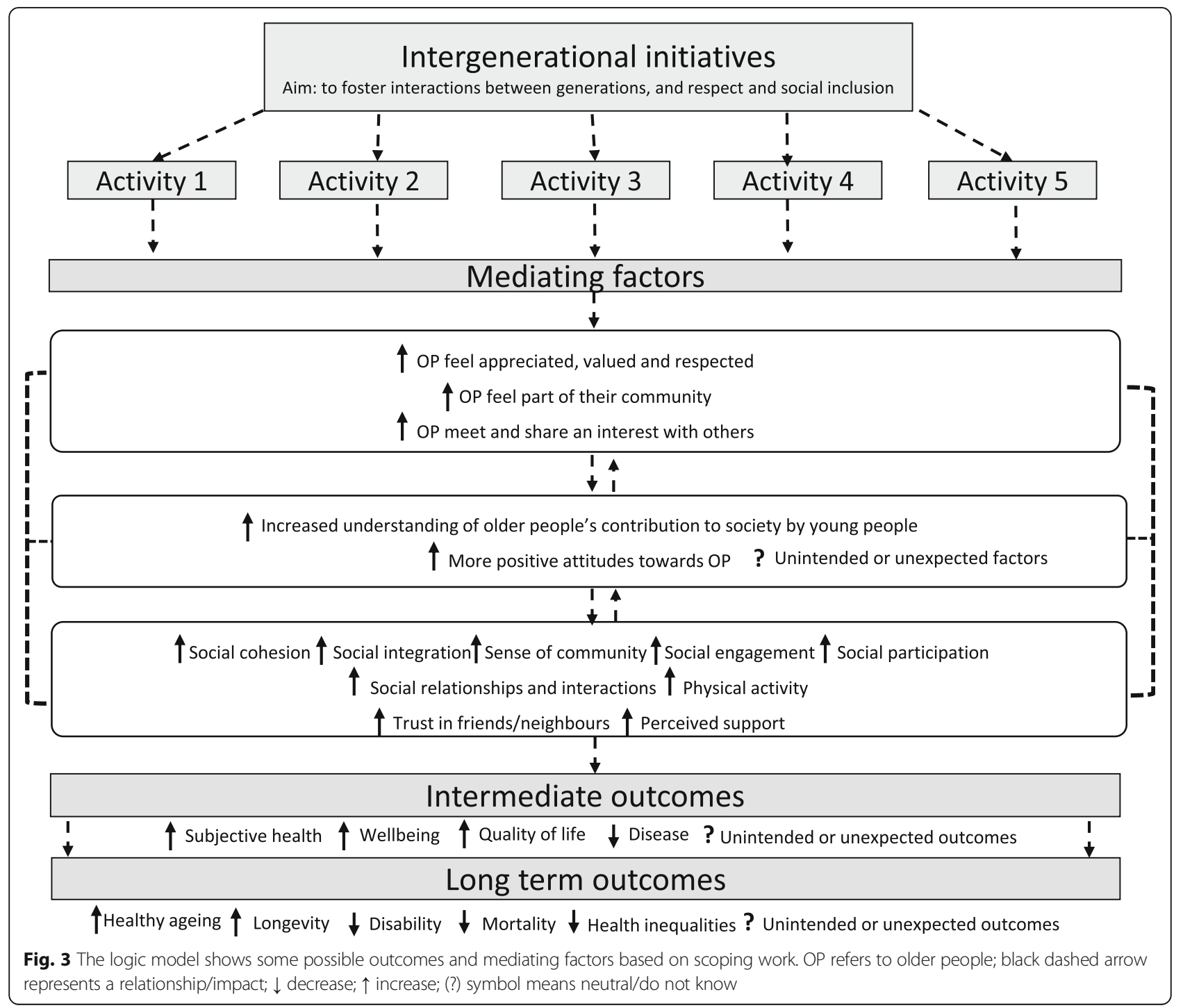




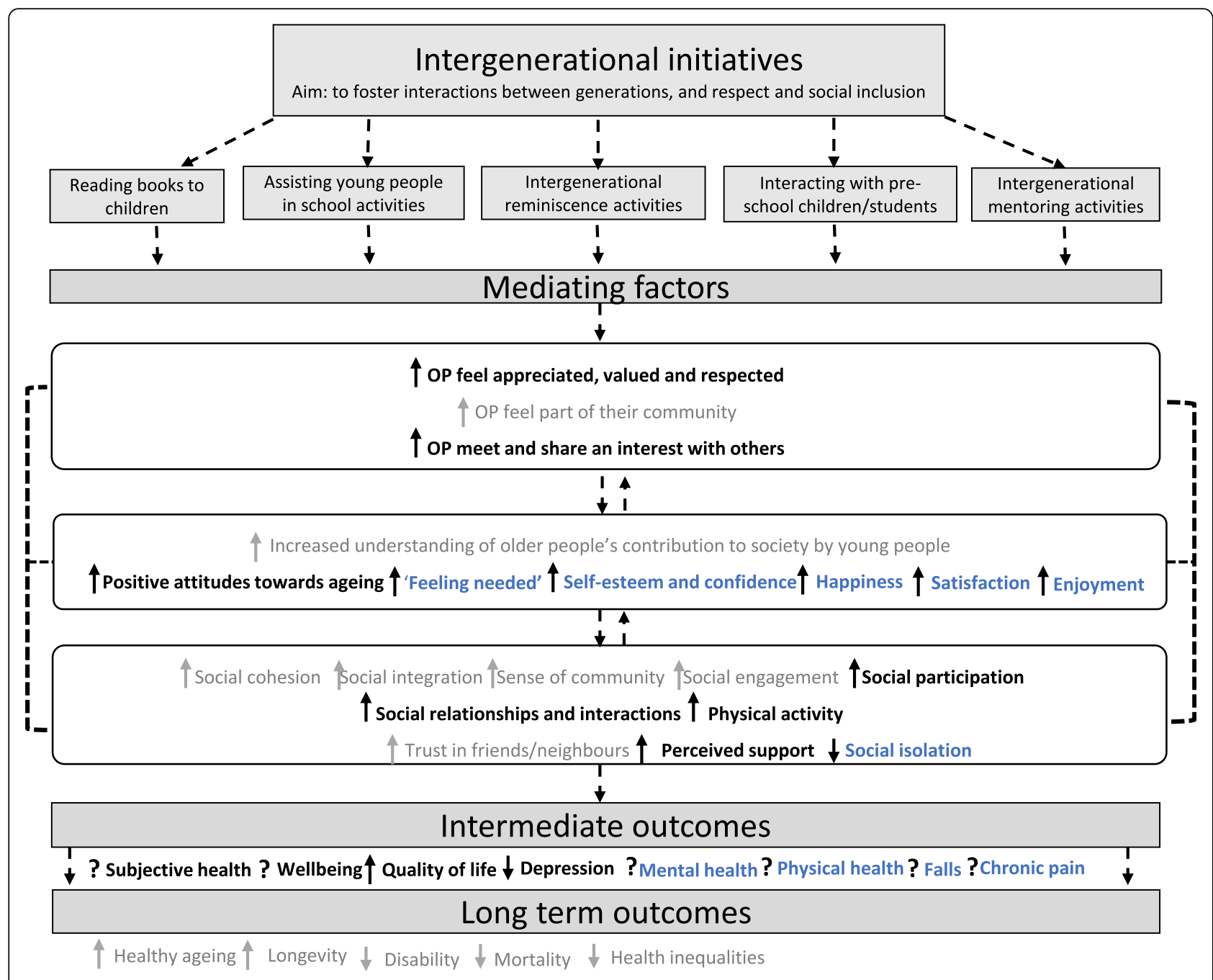

Fig. $4 \mathrm{ln}$ bold are the mediating factors and outcomes that have been studied by the quantitative and/or qualitative studies. In blue are the additional mediating factors and outcomes identified in this review. OP refers to older people; black dashed lines represent a relationship; $\downarrow$ decrease; $\uparrow$ increase; (?) symbol means neutral/do not know/evidence is inconsistent

quantitative and qualitative evidence). For a quick overview of the strength of the quantitative evidence and the RoB of the studies, please refer to the harvest plot (Table 1). For a more detailed explanation of the findings and $\mathrm{RoB}$ for each study, please refer to Additional file 4 (quantitative studies) and Additional file 5 (qualitative studies). The item-level RoB assessment for each study can be found in Additional file 6 (quantitative studies) and Additional file 7 (qualitative studies).

Whilst many studies reported stratification by socioeconomic status, education and gender at baseline, few reported sub-analyses of health outcomes by age, ethnic or education of older people.

\section{Mentoring interventions}

Two quantitative studies looked at mentoring (Additional file 4): an individual RCT of a community-based mentoring service programme rated as low-moderate RoB [77] and an uncontrolled before and after study of an intergenerational mentoring programme rated as high RoB [65]. Differences observed between comparison groups at baseline [77] and small sample sizes [65] made it difficult to interpret the results.

One study found no effect on depressive symptoms (mean difference $(\mathrm{MD})=0.2, p=0.29$ ) [77], and although it showed a significant improvement in subjective health at 6 -month follow-up $(\mathrm{MD}=-0.09, p<0.01)$, this improvement was significantly less than controls $(\mathrm{MD}=-0.1, p<0.01)$ [77]. Two studies found no effect on mental health ( $\mathrm{MD}=0.8, p=0.48$ [77]; $\mathrm{MD}$ and $p$ values not reported in the study by Ellis [65]) and physical health $(\mathrm{MD}=0.1, p=0.90$ [77]; $\mathrm{MD}$ and $p$ values not reported in the study by Ellis [65]). A further study did not observe an effect of mentoring on quality of life 
(MD and $p$ values not reported in the study by Ellis [65]).

One qualitative study included a mentoring programme, where older people acted as mentors for pre-school children [85] (Additional file 5). It included limited reporting of analysis, sampling and results. From the older people's narratives, mentoring children was reported to help participants going through difficult times in their lives and to enhance their physical and mental wellbeing. Reported factors that might lead to an improvement in wellbeing were the following: improved self-esteem, satisfaction, confidence, interactions and relationships and feeling valued (Fig. 5).

\section{Intergenerational interventions}

Intergenerational studies included (i) mentoring initiatives $[68,81]$, (ii) interventions based on service-learning pedagogy [87], (iii) school initiatives [76, 82, 104], (iv) reading initiatives [83, 84], (v) reminiscence initiatives [75] and (vi) interventions involving reading and drawings [103].

Eleven quantitative studies were included Additional file 4): one individual RCT [76], one cluster RCT [81], two cluster controlled trials [83, 84], two controlled before and after studies [82, 87] and five uncontrolled before and after studies $[65,68,75,103,104]$. Seven were judged as lowmoderate RoB, with four being moderate-high RoB [65, 76, $103,104]$. The main weaknesses of these studies were small sample size $(n=2)[103,104]$ and lack of a control group $(n=2)[103,104]$. Reporting of analysis was limited in three studies $[65,76,104]$.
Five studies found a significant effect on depression scores (reduction of $62 \%$ within 2 weeks after the completion of the programme: $\mathrm{MD}=1.86, p$ value not reported in the study [68]; reduction of $26.3 \%$ obtained in the posttreatment evaluation: $\mathrm{MD}=3.53, p<0.001$ [87]; reduction of $18.5 \%$ at 2-year follow-up: $\mathrm{MD}=0.94, p<0.001$ [82]; reduction of $14 \%: \mathrm{MD}=0.31, p<0.10$ [84]; reduction of $16.64 \%$ at 68-week follow-up: MD and $p$ value not reported in the study [104]), whilst one study found no effect at 8-week follow-up ( $\mathrm{MD}=-0.97, p=0.3)$ [103].

One study showed a significant favourable effect on selfrated health scores at 21-month follow-up $(p<0.01$; MD not reported in the study by Fujiwara et al. [83]), whilst one study did not find an effect at 4-month follow-up $(p=0.554)$ [81]. For quality of life scores, two studies showed some indication of an effect (an increase of 4.4\% in the subscale of past, present and future activities after the completion of the programme: $\mathrm{MD}=-0.65, p=0.05$ [75]; an increase of 7\%: $\mathrm{MD}=-1.91, p$ value not reported in the study by Chung [68]). One study (high RoB) did not observe an effect on quality of life, physical health and mental health [65]. In one study, participants experienced a non-significant decrease of more than $50 \%$ in falls rates at 4-8-month follow-up ( $p=0.17)$ [76].

Three qualitative studies, of low-moderate RoB, were included (Additional file 5). Participants' narratives identified some factors mediating the impact of wellbeing, subjective health and depressive $\operatorname{mood}[85,86,89]$ (Fig. 6). These included the following: improved selfesteem and confidence, enjoyment and satisfaction and

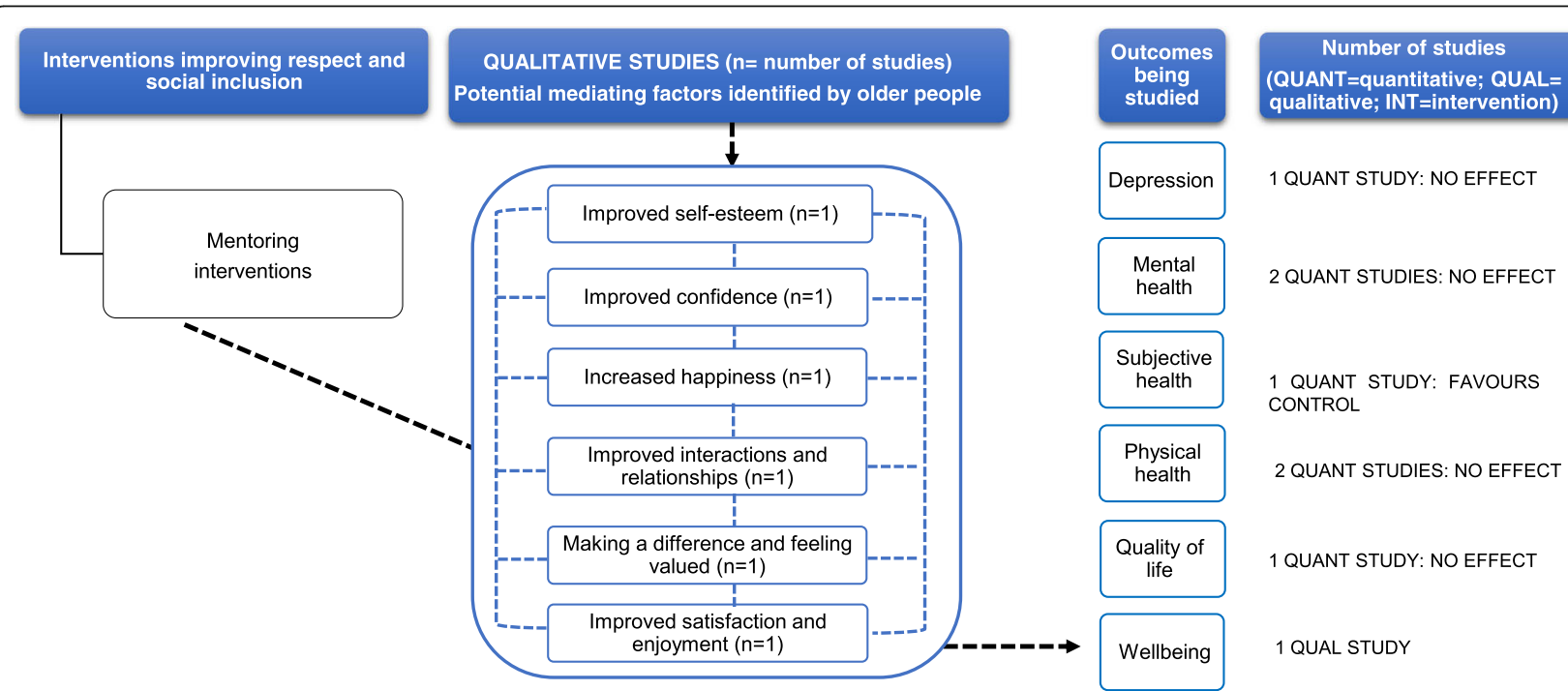

Fig. 5 This diagram shows an overview of the outcomes (depression, mental health, subjective health, physical health, quality of life and wellbeing) that have been studied by the qualitative and quantitative studies (including number of studies), the effect for quantitative studies and the possible mechanisms for these effects as suggested by the qualitative evidence. The dashed arrows that go from the mediating factors to the outcomes indicate solely that according to some participants' narratives, these factors may contribute to an improvement in health outcomes. See Additional files 4 and 5 for a summary of the studies, and the harvest plot (Table 1), which graphically represents the overall summary of the quantity, direction and strength of the quantitative evidence for the various health outcomes 


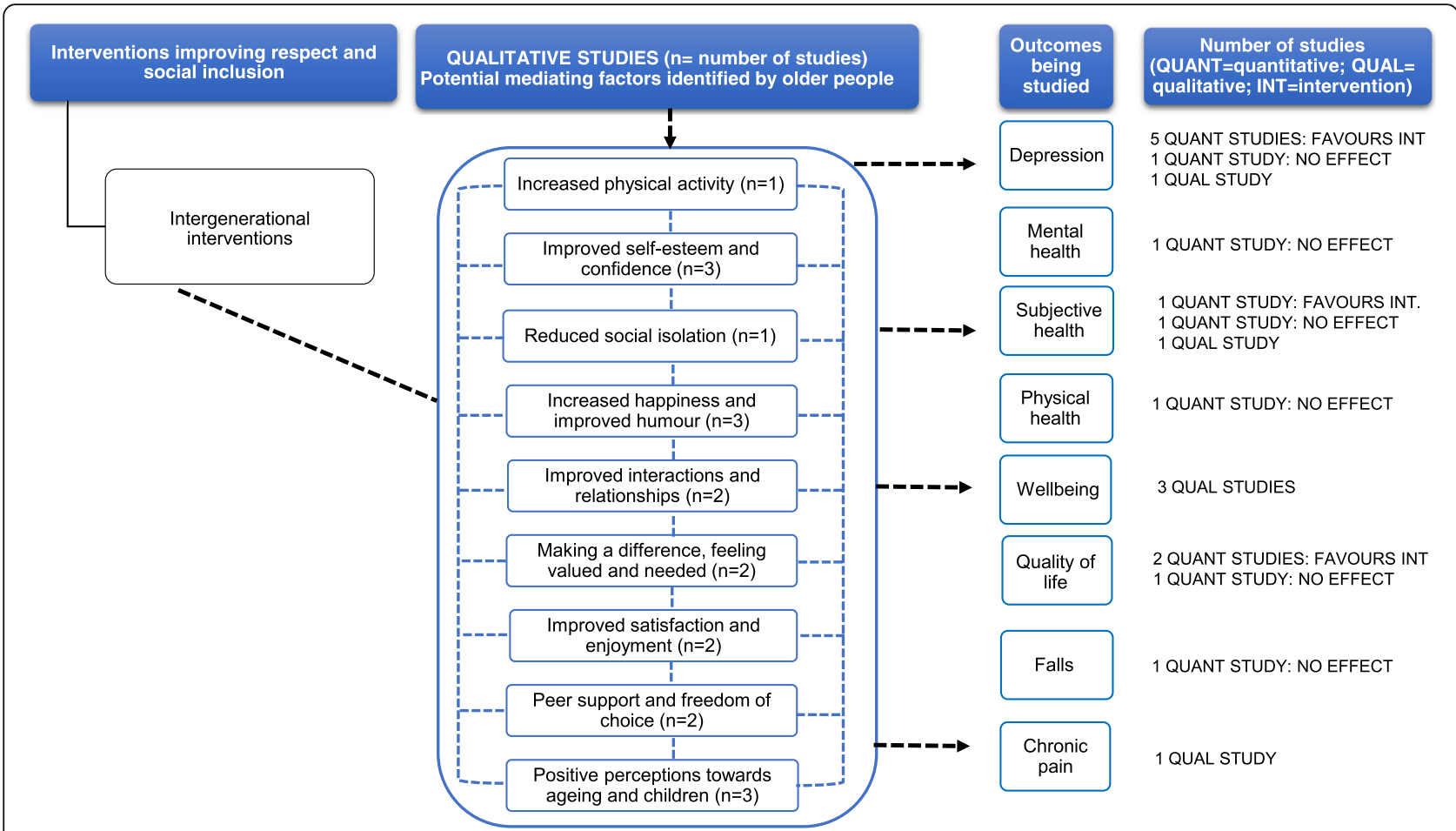

Fig. 6 This diagram shows an overview of the outcomes (depression, mental health, subjective health, physical health, wellbeing, quality of life, falls and chronic pain) that have been studied by the qualitative and quantitative studies (including number of studies), the effect for quantitative studies and the possible mechanisms for these effects as suggested by the qualitative evidence. The dashed arrows that go from the mediating factors to the outcomes indicate solely that according to some participants' narratives, these factors may contribute to an improvement in health outcomes. See Additional files 4 and 5 for a summary of the studies, and the harvest plot (Table 1), which graphically represents the overall summary of the quantity, direction and strength of the quantitative evidence for the various health outcomes

happiness; improved interactions and relationships with others; feeling valued; and positive perceptions towards ageing and children. Older people's narratives reported a perceived enhanced emotional and physical wellbeing and subjective health $[85,86,89]$. In a study conducted by De Souza [86], the female participants reported that the project helped to alleviate their depressive moods and to improve their overall wellbeing and humour.

\section{Dancing interventions}

Two quantitative studies were included (Additional file 4): an individual RCT [71] and an individual controlled trial [70]. They were both rated as high and moderate RoB $[70,71]$ due to differences between control and intervention groups in the depression levels at the outset of the study [71] and small samples [70, 71].

One study showed significant reduction in depression scores [71] (older people with Parkinson's disease: $\mathrm{MD}=0.26, p=0.001$; older people without Parkinson's disease: $\mathrm{MD}=0.52, p=0.001$ ). Neither study found an effect on wellbeing and subjective health between 2-week and 68-month follow-up [70]. Findings were mixed for falls rates, with one study showing a significant reduction in falls (MD and $p$ values not reported in the study by Hackney et al. [71]) and the other showing no effect [70].

Two qualitative studies provided context to the relationship between dancing and subjective and physical health, subjective health and wellbeing [70, 72] (Additional file 5). The main weaknesses of the studies included limited reporting of sampling, analysis and results. Participants' narratives identified some factors mediating the impact of physical health, subjective health and wellbeing, and these (Fig. 7) comprised the following: improved satisfaction, enjoyment and confidence; improved fluency, dynamics of movement and mobility; improved social interactions; and feeling valued. Older people talked about how the programme made them feel better, giving them a sense of wellbeing [72], and made them feel good and capable despite some health difficulties [70].

\section{Music and singing interventions}

There were six quantitative and two qualitative studies that explored the impacts of music $[93,101]$ and singing initiatives $[66,69,91,94,102,108]$. The six quantitative studies included (Additional file 4) the following: a cluster RCT [107], an individual RCT [66], a controlled before and after study [93] and three before and after 


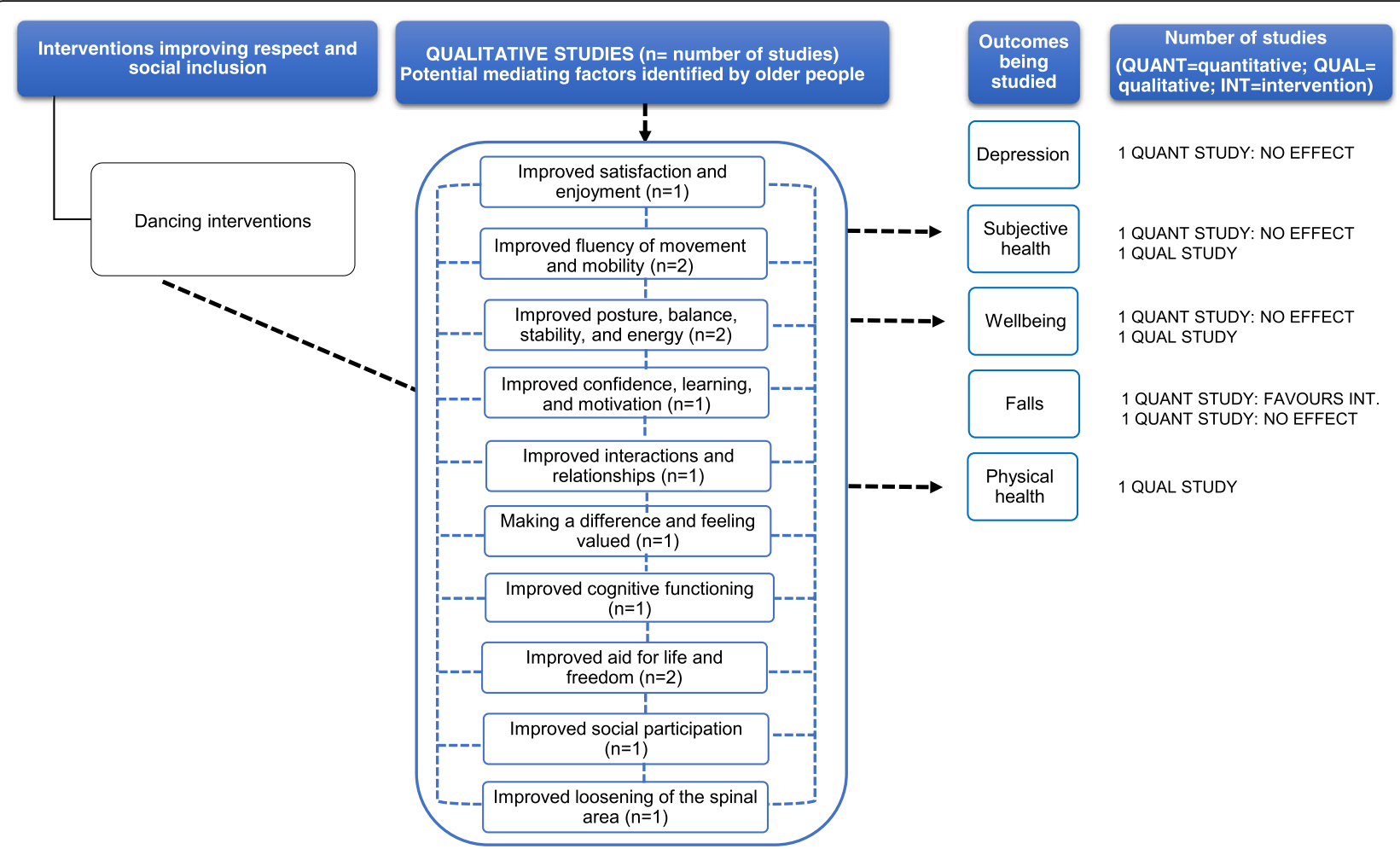

Fig. 7 This diagram shows an overview of the outcomes (depression, subjective health, wellbeing, falls and physical health) that have been studied by the qualitative and quantitative studies (including number of studies), the effect for quantitative studies and the possible mechanisms for these effects as suggested by the qualitative evidence. The dashed arrows that go from the mediating factors to the outcomes indicate solely that according to some participants' narratives, these factors may contribute to an improvement in health outcomes. See Additional files 4 and 5 for a summary of the studies, and the harvest plot (Table 1), which graphically represents the overall summary of the quantity, direction and strength of the quantitative evidence for the various health outcomes

uncontrolled studies $[69,92,94]$. Three were judged as low-moderate RoB [66, 92, 107], two as moderate RoB $[93,94]$ and one as high RoB [69]. The main issues were short follow-up [69, 94], small sample size $[69,94]$ and poor adjustment for potential confounders [93].

With regard to psychological outcomes, one study [91] found a significant reduction of $36.6 \%$ in depression scores at 3-month follow-up ( $\mathrm{MD}=-1.52, p<0.01)$ and of $12.5 \%$ at 6-month follow-up $(\mathrm{MD}=-0.53, p=014)$. The same study [91] found a significant reduction of $31.1 \%$ in anxiety scores at 3 -month follow-up $(\mathrm{MD}=-1.78, p<0.01)$. Two studies showed no effect on reduction in depressive symptoms at 12-month [66] and 8-week [94] follow-up. One study showed a reduction of $27.3 \%$ in perceived stress scores $(\mathrm{MD}=2.58, p<0.001)$ [92].

Two studies found a positive effect on mental health. One study showed a significant improvement of $9.4 \%$ in mental health-related quality of life scores $(\mathrm{MD}=4.77, p<0.01)$ at 3 -month follow-up and of $5 \%$ at 6-month follow-up ( $\mathrm{MD}=2.35 p=0.05)$. Another study found an improvement of $14.3 \%$ in mental health scores (vitality subscale: $\mathrm{MD}=10.4, p=0.03$ ) at 8-week follow-up [94].
Two studies found a positive effect on physical health. One study showed an improvement of $14.3 \%$ in the vitality subscale (vitality subscale: $\mathrm{MD}=10.4, p=0.03$ ) at 8-week follow-up [94]. Another study found an increase of $9.03 \%$ in physical health scores $(\mathrm{MD}=0.72, p<0.01)$ [66] at 12-month follow-up.

For quality of life and wellbeing, results were mixed: one study [93] found an improvement in two components of the wellbeing and quality of life scale (an increase of $14 \%$ in control: $\mathrm{MD}=1.15, p=0.0001$; an increase of $7.6 \%$ in pleasure: $\mathrm{MD}=0.8, p=0.0001$ ) at 9-month follow-up, and the other study found no effect [69]. One study showed a significant reduction of $104 \%$ in falls rates $(\mathrm{MD}=-0.32$, $p<0.05$ ) [66] at 12-month follow-up.

Two qualitative studies at low-moderate RoB gave context to the relationship between singing and music initiatives and the health outcomes [102, 105] (Additional file 5). Older people reported that music-making activities resulted in a better quality of life (e.g. enjoyment), mental health benefits (e.g. ability to cope effectively with stress) and physical health (e.g. good for asthma and breathing) [102, 105]. Participants' narratives identified some factors mediating the impact of various health outcomes (depression, anxiety, 
perceived stress, mental health, physical health, wellbeing and quality of life). These included improved confidence, concentration and sense of achievement; feeling valued; and improved interactions with others (Fig. 8).

\section{Information-communication technology interventions}

Three quantitative studies were included (Additional file 4): two individual RCTs [67, 95] (low and moderate RoB) and one controlled before and after study [78] (moderate-high RoB).

Three studies found a non-significant reduction in depression scores $(\mathrm{MD}=-1.4, p=0.56[67] ;-0.12$ decrease on a $0-15$ scale, $p$ value not reported in the study by Woodward et al. [95]; 0.2 increase on a $0-15$ scale, $p$ value not provided in the study by Woodward et al. [78]). One study [67] found non-significant reduction in anxiety scores $(\mathrm{MD}=-0.25, p$ $=0.56)$, improvement in mental health $(\mathrm{MD}=1.03, p=0.10)$ and physical health $(\mathrm{MD}=2.63, p=0.14)$. Findings were mixed for the two studies looking at quality of life outcome scores, with one intervention showing an improvement (4.99 increase on a 16-112 scale, $p<0.05$ ) [95] at 6-month follow-up and the other showing no effect (6.1 increase on a
16-112 scale, $p$ value not provided in the study by Woodward et al. [78]).

One qualitative study (moderate RoB) [80] reported a perceived improvement in wellbeing (Additional file 5). Study participants related their enhanced sense of wellbeing acquired from using information-communication technology (ICT) to an increased sense of purpose and enjoyment to their lives. Some older people reported the programme served as a medium for strengthening existing relationships. Others mentioned that having ICT as a common interest brought them closer to family members. Other factors mediating the impact of wellbeing included improved health maintenance, satisfaction, civic engagement and feeling valued (Fig. 9).

\section{Art and culture interventions}

Five quantitative studies were included (Additional file 4): one individual controlled trial [66] and four before and after uncontrolled studies [74, 96, 98, 109]. Studies were rated as low-moderate $\operatorname{RoB}(n=2)[66,96]$, moderate $\operatorname{RoB}$ $(n=2)[97,98]$ and high-moderate $\operatorname{RoB}(n=1)$ [74]. Study weakness included small sample size, no control group and adjustment for known confounders not reported.

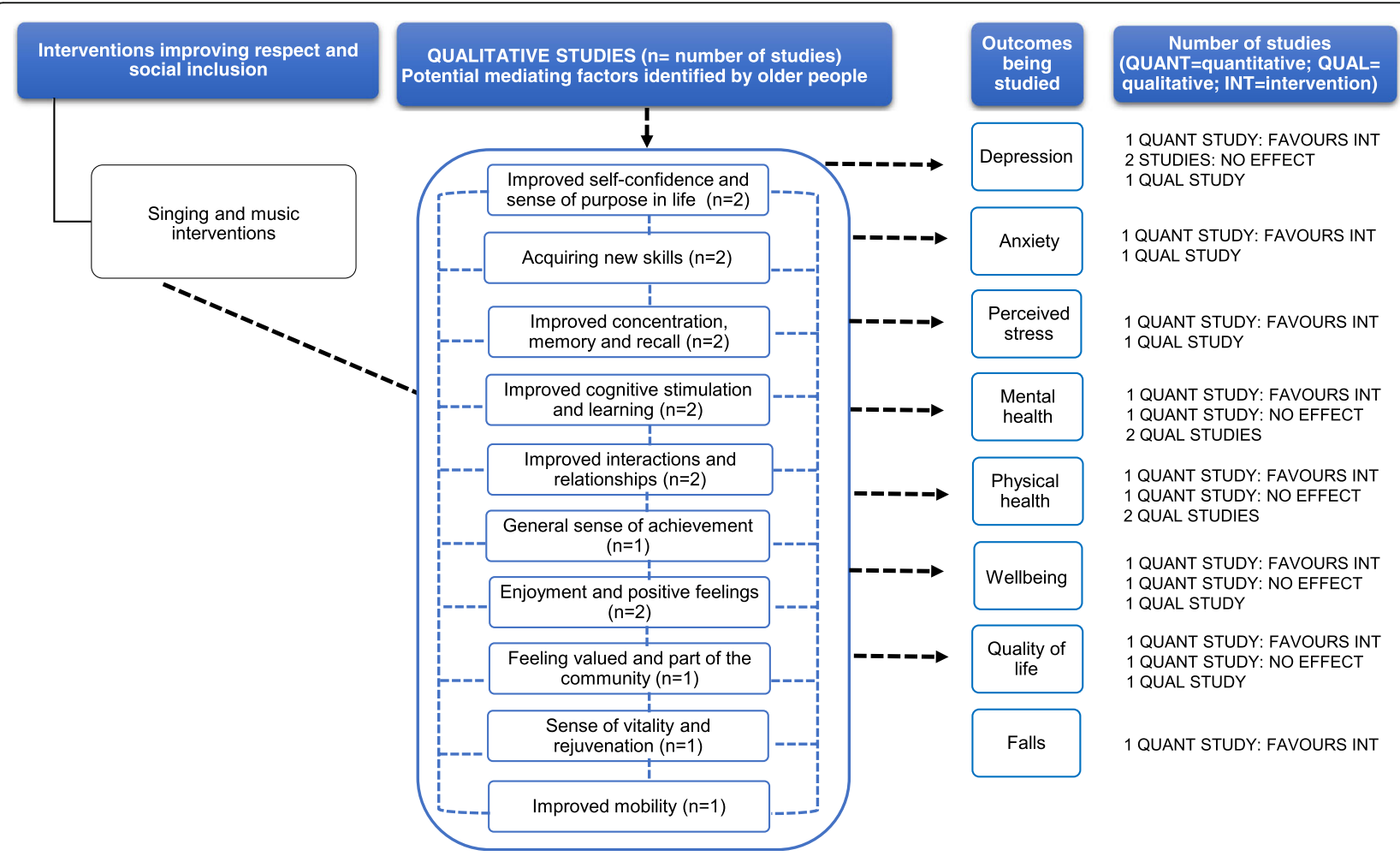

Fig. 8 This diagram shows an overview of the outcomes (depression, anxiety, perceived stress, mental health, physical health, wellbeing, quality of life and falls) that have been studied by the qualitative and quantitative studies (including number of studies), the effect for quantitative studies and the possible mechanisms for these effects as suggested by the qualitative evidence. The dashed arrows that go from the mediating factors to the outcomes indicate solely that according to some participants' narratives, these factors may contribute to an improvement in health outcomes. See Additional files 4 and 5 for a summary of the studies, and the harvest plot (Table 1), which graphically represents the overall summary of the quantity, direction and strength of the quantitative evidence for the various health outcomes 


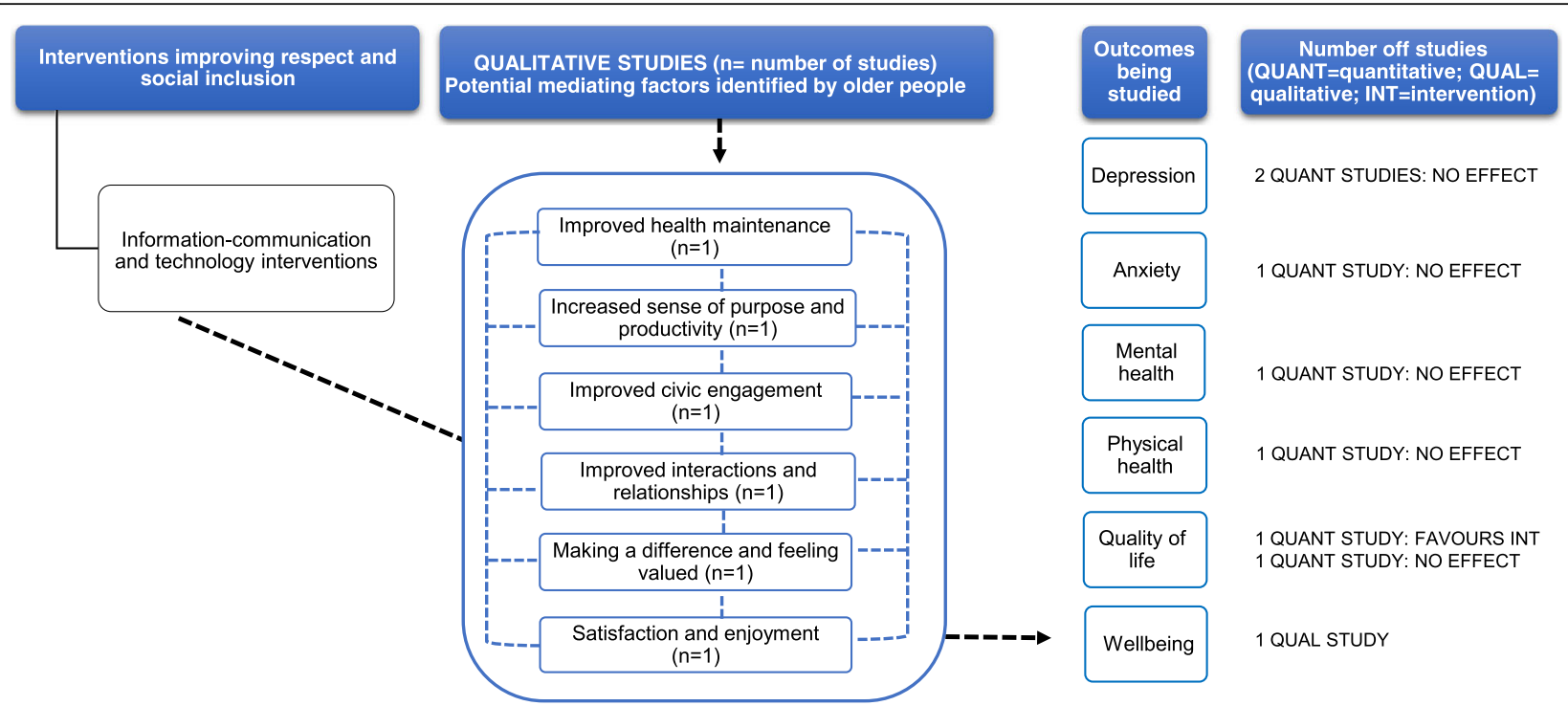

Fig. 9 This diagram shows an overview of the outcomes (depression, anxiety, mental health, quality of life and wellbeing) that have been studied by the qualitative and quantitative studies (including number of studies), the effect for quantitative studies and the possible mechanisms for these effects as suggested by the qualitative evidence. The dashed arrows that go from the mediating factors to the outcomes indicate solely that according to some participants' narratives, these factors may contribute to an improvement in health outcomes. See Additional files 4 and 5 for a summary of the studies, and the harvest plot (Table 1), which graphically represents the overall summary of the quantity, direction and strength of the quantitative evidence for the various health outcomes

With regard to psychological outcomes, two studies showed non-significant reductions in depression scores at 2-year follow-up $(\mathrm{MD}=0.7)[96]$ and at 12-week follow-up $(\mathrm{MD}=-0.7)[66]$. One study showed no effect on mental health at 1-month follow-up (MD $=-2.8, p=0.154$ ) [98].

One study found a significant improvement of $21.1 \%$ in physical health scores (MD $=-11.9, p=0.030)$ [98] at 1-month follow-up. Two studies found a significant effect on subjective health scores (an increase of 14\% $(\mathrm{MD}=-0.4, \quad p<0.10)$ [96] at 2-year follow-up; an increase of $9 \%(\mathrm{MD}=0.72, p<0.01)$ [66] at 12-week follow-up).

In terms of wellbeing scores, one study found a significant effect (an increase of 27.6\%: $\mathrm{MD}=-20.2, p=0.002$ ) [98], and one found no effect $(\mathrm{MD}=-6)$ [74]. One study did not find an effect on health-related quality of life scores $(\mathrm{MD}=$ not reported, $p=0.88)$ [97]. One study showed a significant reduction in falls rate (reduction of $104 \%, p<0.05$ ) [66] and another on chronic pain scores (reduction of 23\%: $\mathrm{MD}=0.5, p<0.05$ ) [96].

Three qualitative studies were included (Additional file 5). Participants' narratives provided context to the association of art and culture interventions with health outcomes (depression, anxiety, perceived stress, wellbeing, quality of life and chronic pain). Older people described how creative work helped them to reduce their feelings of stress and anxiety and to overcome some health limitations (e.g. depression) [96, 110]. They also reported feeling more socially and physically active and feeling more relaxed [96].
Other factors mediating the impact included reduced social isolation, increased self-confidence, social connectedness, improved social interactions and feeling valued (Fig. 10).

\section{Multi-activity interventions}

Five quantitative studies were included: an individual RCT [99], two individual controlled trials [73, 79] and two before and after uncontrolled studies [100, 106] (Additional file 4). Studies were rated as low to moderate $\operatorname{RoB}(n=3)$ [79, 99, 106], moderate $\operatorname{RoB}(n=1)$ [100] and moderate-high RoB $(n=1)$ [73]-due to no random allocation of the intervention or control groups and convenience sampling methods.

Multi-activity interventions included (i) projects to encourage older people to participate in various activities organised in the city [99], (ii) creative exercise and/or cultural activities wherein older people were guided by peers [100], (iii) regular gatherings in neighbours' homes and interactions with others [106], (iv) social clubs and exercise programmes $[79,90]$ and (v) regular meetings to discuss health information topics including people's feelings and health [73].

Findings for psychological outcomes were mixed. One study found a significant reduction of $13.4 \%$ in depression scores at 6-month follow up (MD $=0.60, p<0.02)[100]$ and of 11.6\% at 12-month follow-up ( $\mathrm{MD}=0.56, p<0.05)$ [100]. By contrasts, two studies did not find an effect $(\mathrm{MD}=0.03$ at 9-month follow-up [106]; MD $=0.4$ at 6-month followup [99]). One study showed a significant improvement of 


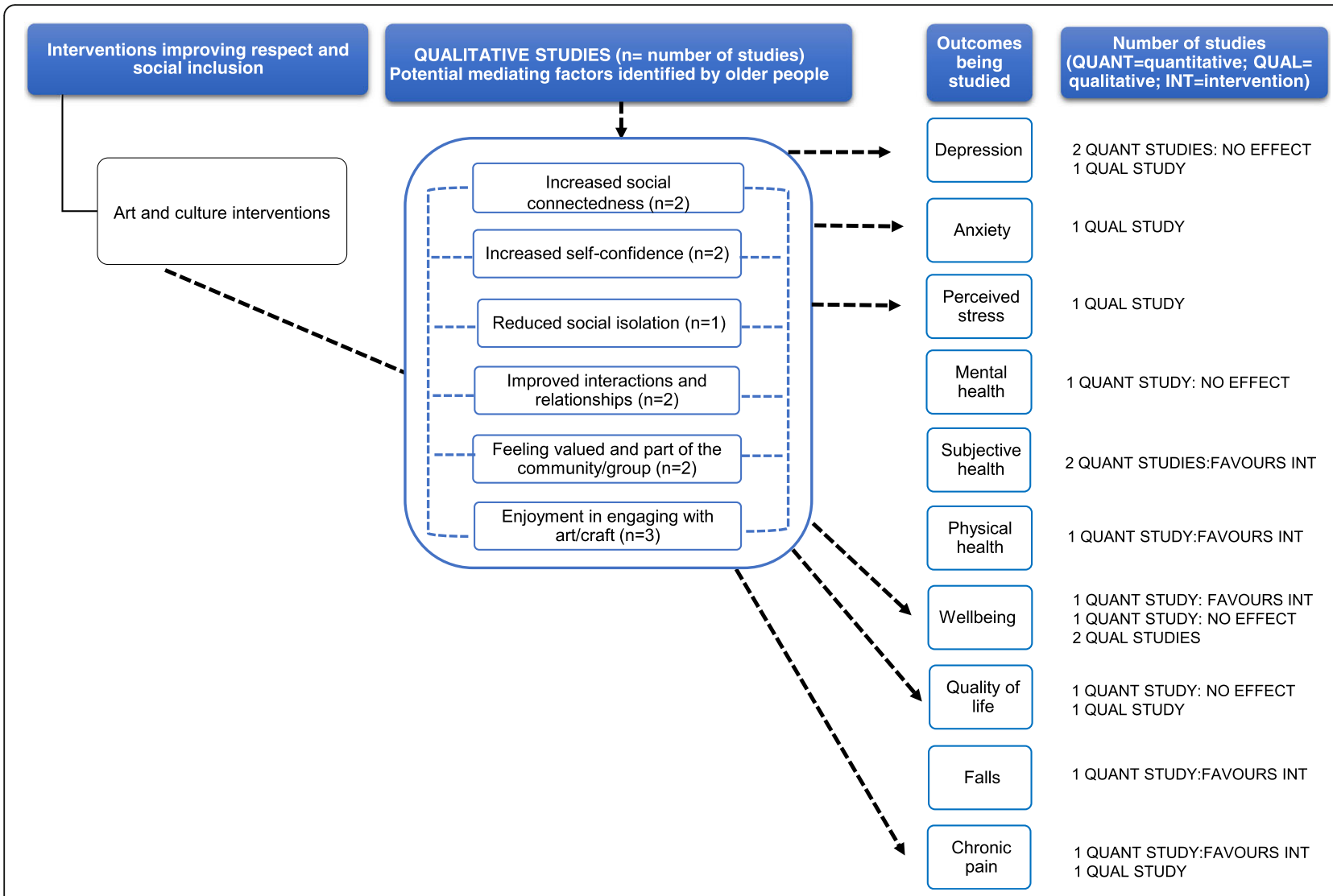

Fig. 10 This diagram shows an overview of the outcomes (depression, anxiety, perceived stress, mental health, subjective health, physical health, wellbeing, quality of life, falls and chronic pain) that have been studied by the qualitative and quantitative studies (including number of studies), the effect for quantitative studies and the possible mechanisms for these effects as suggested by the qualitative evidence. The dashed arrows that go from the mediating factors to the outcomes indicate solely that according to some participants' narratives, these factors may contribute to an improvement in health outcomes. See Additional files 4 and 5 for a summary of the studies, and the harvest plot (Table 1), which graphically represents the overall summary of the quantity, direction and strength of the quantitative evidence for the various health outcomes

$6.24 \%$ in mental health scores $(\mathrm{MD}=3, p<0.005)[100]$ but at the first follow-up only (6 months). One study found a significant reduction of $11.7 \%$ in perceived stress scores ( $\mathrm{MD}=2.23, p<0.001$, at 9-month follow-up) [106]

Two studies found a positive effect on subjective health scores (an increase of 5.15\% $(\mathrm{MD}=0.37, p<0.01)$ at 3-month follow-up [79]; an increase of $4.2 \%(\mathrm{MD}=1.57$, $p=0.06$ ) at 12-month follow-up [100]). One study found a positive effect on wellbeing scores (an increase of 9\%: $\mathrm{MD}=-1.9, p=0.039$ [99]) at 6-month follow-up. By contrast, two studies did not find an effect on wellbeing $(\mathrm{MD}=0.42$ [79]; $\mathrm{MD}=1.47$ [73]).

Two qualitative studies were included (Additional file 5). Participants' narratives gave insight on the relationship between multi-activity interventions and reduction in depression [100], wellbeing [90] and improved physical health [90, 100] (Fig. 11). These included (i) improved attention to self-care, self-worth and enjoyment; (ii) improved social interactions; and (iii) and feeling valued. Older people reported perceived psychological and physical health benefits including feeling better, increased flexibility and strength.

\section{Discussion}

This is the first systematic review to report on the health impacts of interventions promoting respect and social inclusion in community-residing older people. By drawing on data from both quantitative and qualitative studies, it uniquely furthers our understanding of the pathways that may lead to improved health and wellbeing.

\section{Summary of findings}

Intergenerational and music and singing interventions (for which there was the largest evidence base: 14 studies for intergenerational initiatives and eight studies for singing and music interventions), art and culture and multiactivity interventions showed an overall positive effect on various health outcomes. Quantitative studies identified impacts on depression $(n=3)$, wellbeing $(n=3)$, subjective health $(n=2)$, quality of life $(n=2)$, perceived stress and 


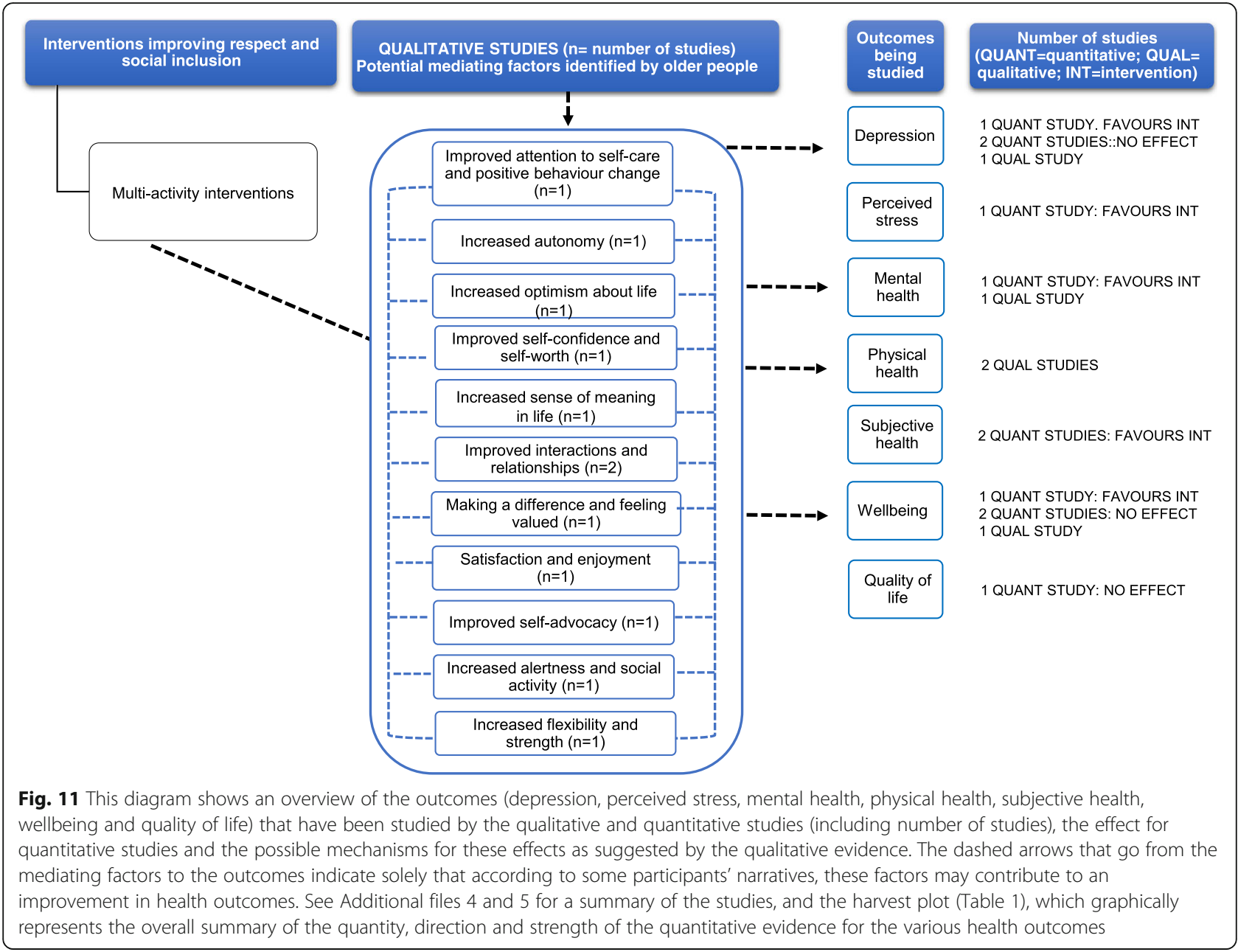

mental health $(n=2)$ and physical health $(n=2)$. In contrast, due to a paucity of evidence for mentoring, dancing and ICT interventions, it was not possible to make a judgement of the impact on health outcomes.

Qualitative studies identified some mediating factors (e.g. improved self-esteem) that may lead to improvements in health outcomes. For instance, in most intergenerational initiatives (Fig. 6), older people were regularly involved in assisting young people in school activities (e.g. math problems), and reading books to pre-school children. It appears that regular interaction with young people may have led older people to feel more valued, included, and appreciated. As a result, older people reported enhanced subjective health.

\section{Findings in relation to the literature}

A number of reviews have explored the links between different social aspects of ageing and health outcomes [111-119]. For instance, the Centre for Reviews and Dissemination (CRD) [120] has provided a summary of several systematic reviews of interventions addressing social isolation and loneliness in older people [111-116].
Although related, social isolation and loneliness differ from the concept of social inclusion adopted in this study. Social isolation mainly refers to the quantity and quality of social support or contact received by others [111]. The same applies for loneliness, which is defined as "a subjective concept resulting from a perceived absence or loss of companionship" [111]. Dickens et al. [111] looked at both one-to-one and group-based interventions targeting social isolation and loneliness. They found that group-based interventions (e.g. psychosocial activity group) were more likely to have a positive effect on at least one of the four social health subdomains if compared with the one-to-one interventions (e.g. volunteer home visiting intervention). As we were interested in interventions focusing on making people valued and part of the community, we included only group-based interventions.

Previous reviews have looked at health impacts of specific interventions, including music [121], computer and internet training [116], dancing [122, 123] and genderbased interventions [117]. In her scoping review, Milligan et al. [117] assessed the evidence for the impacts of gendered social interventions (Men's Sheds) on the health and 
wellbeing of older men. There are some similarities with our review, in terms of the complexity and typology of interventions as well as the approach used to synthesise the evidence of these studies. Firstly, although very specific, Men's Sheds interventions aim to encourage older men to meet and socialise, learn new skills and take place in a community setting. Secondly, Milligan et al. [117] included qualitative and quantitative evidence and found that interventions were heterogeneous particularly in terms of (i) methodology, (ii) outcome measures and (iii) variety of activities within the interventions. Thirdly, the main weakness of their studies included low sample representativeness and lack of control group. All these aspects contributed to challenges in synthesising evidence of the health benefits of these interventions, as in our review.

Other reviews have focused on a diverse range of interventions but examined the effect on specific health outcomes. For instance, Lafortune et al. [118] examined the effectiveness and cost-effectiveness of various interventions promoting healthy behaviours (e.g. diet, physical activity/inactivity, alcohol, smoking, cognitive activity and risk reduction relating to loneliness and isolation) and their impact on primary prevention or delay of cognitive decline or dementia. They reported that interventions promoting social participation were associated with an overall positive impact on cognitive outcomes. Similar to our review, they found that reading to children in schools or art sessions may improve social, mental or physical health of older people. Disadvantaged groups were also underrepresented, with many studies being heterogeneous in intervention types and/or outcome measures.

The scope of our review includes all types of interventions that aimed to improve respect and social inclusion in older people and assessed associated impacts on health and wellbeing. Only six studies [66, 67, 71, 76, 83, 95], included in the reviews mentioned above $[111,115,116$, $118,119,121,123]$, were directly concerned with older people and with the definition of interventions promoting respect and social inclusion adopted in this study.

\section{Strengths and limitations}

We adopted a comprehensive and systematic approach for reviewing the evidence on a complex topic. All study designs were considered, and our inclusive approach allowed us to include a range of intervention types and health outcomes and positive and negative effects, which we attempted to summarise in the harvest plot (Table 1). The search was restricted to studies published in English, and this may have introduced language bias since significant results are more likely to be published in English-language journals than those reporting non-significant results [124]. This may also explain why all included studies concerned higher and upper middle-income countries. Due to the heterogeneity of the included studies, we used a narrative synthesis approach to summarise the findings of studies of this review. We were therefore unable to quantitatively assess publication bias by, for example, looking for funnel plot asymmetry [40].

We used the LQATs to assess the RoB of quantitative studies. LQATs have been used in a number of previous systematic reviews $[125,126]$ and have been critically examined in relation to other quality appraisal tools [127]. Qualitative studies were appraised using established criteria related to reliability and validity of findings developed by Harden et al. [62] and Mays and Pope [63]. Whilst these tools have been used extensively, the global assessment approach that we used was not previously validated in the appraisal of the original tools. An important limitation of this systematic review is that the majority of the review work was conducted by one reviewer, and some eligible studies may have been missed [40].

By drawing on both quantitative and qualitative evidence, we have explored both the effectiveness of relevant interventions (primarily quantitative evidence) and the mediating factors to improve health and wellbeing outcomes (primarily qualitative evidence). We feel that this approach has led to a better overall understanding of the current evidence base on interventions on respect and social inclusion in older people than would not have been possible using either quantitative or qualitative evidence alone [35, 128]. Qualitative studies helped us to understand some of the complexity of the wide range of components of each intervention and to clarify some aspects of the complexity related to how and why interventions may work or not work $[29,46,128]$. By doing so, qualitative studies contributed to the assessment of causality.

\section{Public health and policy implications}

Many of the interventions reviewed were delivered as projects to selected groups, raising important questions about feasibility of wider implementation and potential for population benefits $[129,130]$. Our findings suggest that studies mainly relied on people who volunteered. Since these people are generally more willing to participate in the community, they may not be representative of the entire population, particularly of hard-to-reach older people (e.g. those experiencing social exclusion, isolation, poverty and health problems). Services and other initiatives promoting respect and social inclusion (and similar approaches) should be provided to every older person who stands to benefits from these, and good policies in place should remove the barriers that limit people in most need (e.g. marginalised groups) in accessing these interventions [4].

\section{Research implications}

Many of the interventions included in this review were implemented through weekly and monthly activities (e.g. 
reading books to children). These activities were facilitated by professionals, students, peers or older people themselves and took place in community centres and schools. Further research should assess the cost-effectiveness of these interventions (including when applied at greater scale in response to population need), particularly those that have shown a positive health impact (singing and music, intergenerational interventions, art and culture and multi-activity interventions).

Whilst age, gender, education, ethnic and socioeconomic status of older people were recorded in the data extraction tables, only two studies reported them, and overall, the quantity and heterogeneity of the evidence precluded useful analysis of differential effects. Newman et al. [104] explored the effect of an intergenerational programme in reducing perceived depression by education level and age. The study showed that older people in the lower education group (high school) experienced an increase of $1.61 \%$ in perceived depression at 6-8-week follow-up. By contrast, those in the higher education group (college) reported a decrease of $26.42 \%$ in perceived depression at 6-8-week follow-up. About the effects by age, the older group (70 and over) experienced a decrease of $24.27 \%$ in perceived depression at $6-8$ weeks post test, whilst the younger group (60 and over) reported an increase of $4.77 \%$ in perceived depression at 6-8 weeks post test (Additional file 4). One qualitative study [86] has reported differences in perceived impacts between males' and females' narratives, such that whilst male and female participants reported an improvement in subjective health, only females reported that the project helped them to alleviate their depressive moods and to improve their overall wellbeing and humour. Looking at differential effects would be a potentially important topic for future analyses as the evidence base expands.

Fifteen studies lacked a control group, making it difficult to be confident that self-reported improvements in psychological outcomes, subjective health, wellbeing and quality of life were directly attributable to the actual interventions. When interpreting our findings, we should note that some studies may have shown a favourable effect as a result of the Hawthorne effect, whereby participants' awareness of being observed may have engendered beliefs about researcher expectations [131].

Considering these challenges, more robust evidence is needed to provide more certain/significant answers about the impact of these interventions. Future studies should (i) take advantage of natural policy experiments fostering respect and social inclusion, (ii) design better in-depth qualitative studies to explore the influence of context and mediating factors, (iii) use rigorous methodologies including randomised designs and (iv) assess whether the most promising interventions are also the most cost-effective.

\section{Conclusions}

In the context of an increasing ageing population, it is important to establish what is known about the impacts of interventions that have the potential to improve older people's health. This review suggests that music and singing, intergenerational initiatives, art and culture and multi-activity interventions may positively impact on wellbeing, subjective health, quality of life and physical and mental health. From the qualitative studies, there was evidence of plausible mediating factors including strengthened social relationships, improved selfconfidence and self-esteem, feeling valued, reduction of social isolation and feeling more physically active. However, the evidence is based on studies with heterogeneous methodologies. Many of the interventions were delivered as projects to selected groups, raising important questions about the feasibility of wider implementation and the potential for population-wide benefits. Future studies which explore potential effect modifiers and mediators will help to strengthen the evidence base and assess whether interventions have the potential to reduce health inequalities.

\section{Additional files}

\begin{abstract}
Additional file 1: PRISMA 2009 checklist. PRISMA 2009 checklist. (DOC 66 kb)
Additional file 2: Search strategy database(s): Ovid MEDLINE(R) and Ovid OLDMEDLINE(R). Search strategy MEDLINE. (DOCX 14 kb)

Additional file 3: Overview of the health outcomes and scales used to assess the interventions on respect and social inclusion (34 studies in total). Overview of the health outcomes and scales used to assess the interventions on respect and social inclusion. (DOCX $20 \mathrm{~kb}$ )
\end{abstract}

Additional file 4: Summary of the quantitative evidence of the included studies stratified by intervention type. Summary table for quantitative studies [132-135]. (DOCX 94 kb)

Additional file 5: Summary of the qualitative evidence of the included studies stratified by intervention type. Summary table for qualitative studies [136]. (DOCX $42 \mathrm{~kb})$

Additional file 6: Item-level risk of bias (RoB) assessment for quantitative studies using the Liverpool University Quality Assessment Tool (LQAT) (Pope [61]). Item-level risk of bias assessment for quantitative studies. (DOCX $39 \mathrm{~kb})$

Additional file 7: Item-level risk of bias (RoB) assessment for qualitative studies using tools adapted from Harden et al. [62] and Mays and Pope [63]. Item-level risk of bias (RoB) assessment for qualitative studies. (DOCX 23 kb)

\section{Abbreviations}

PRISMA: Preferred Reporting Items for Systematic Reviews and MetaAnalyses; WHO: World Health Organization

\section{Acknowledgements}

We thank Mark Petticrew for his valuable comments on the earlier drafts of this manuscript, and Andy Pennington for his technical assistance with EPPIReviewer 4 software.

\section{Funding}

This doctoral research was supported by the National Institute for Health Research School for Public Health Research (NIHR SPHR) (Grant number: SPHR-SWP-AWP-WP4). The views expressed are those of the author(s) and not necessarily those of the NHS, the NIHR or the Department of Health. 


\section{Availability of data and materials}

N/A-No primary data collected.

\section{Authors' contributions}

SR, LO, DP and NB developed the idea for the article. SR developed a protocol under the supervision of LO, DP and NB. NKV assisted with the data acquisition and extraction. SR wrote the first draft, with all authors contributing to critical revision of the manuscript for important intellectual content. All authors read and approved the final manuscript.

\section{Ethics approval and consent to participate}

N/A-No primary data collected

\section{Consent for publication}

N/A—No primary data collected

\section{Competing interests}

The authors declare that they have no competing interests.

\section{Publisher's Note}

Springer Nature remains neutral with regard to jurisdictional claims in published maps and institutional affiliations.

\section{Author details}

'Department of Public Health and Policy, University of Liverpool, Liverpool, UK. ${ }^{2}$ Institute for Ageing, Newcastle University, Newcastle upon Tyne, UK.

\section{Received: 4 June 2017 Accepted: 12 January 2018}

\section{Published online: 30 January 2018}

\section{References}

1. United Nations Department of Economic and Social Affairs Population Division. World population, ageing. New York; 2015. doi:ST/ESA/SER.A/390

2. World Health Organization. Measuring the age-friendliness of cities: A guide to using core indicators. Geneva; 2016. http://www.who.int/kobe_centre/ publications/AFC_guide/en/. Accessed 2 Oct 2017

3. Rechel B, Grundy E, Robine J. Ageing in the European Union. Lancet. 2013; 6736:1-11.

4. World Health Organization. The world report on ageing and health. Luxembourg; 2015. https://doi.org/10.1093/geront/gnw037.

5. Beard JR, Bloom DE. Towards a comprehensive public health response to population ageing. Lancet. 2015;385:658-61. https://doi.org/10.1016/S0140 6736(14)61461-6.

6. Government Office for Science. Future of an ageing population. 2016 https://www.gov.uk/government/publications/future-of-an-ageingpopulation. Accessed 2 Oct 2017

7. World Health Organization. Global strategy and action plan on ageing and health (2016-2020). 2017. http://who.int/ageing/global-strategy/en/. Accessed 2 Oct 2017

8. Buffel T. Researchineg age-friendly communities researching age-friendly communities stories from older people as co-investigators. 1st ed. Manchester: The University of Manchester Library; 2015.

9. Mahmood A, Keating N. Towards inclusive built environments for older adults. In: Scharf T, Keating N, editors. From exclusion to Incl. old age: Policy; 2012

10. World Health Organization. Global age-friendly cities: a guide. Geneva; 2007. http://www.who.int/ageing/age_friendly_cities_guide/en/. Accessed 2 Oct 2017

11. Buffel T, Phillipson C, Scharf T. Experiences of neighbourhood exclusion and inclusion among older people living in deprived inner-city areas in Belgium and England. Ageing Soc. 2012;33:89-109. https://doi.org/10.1017/ S0144686X12000542.

12. Ronzi S, Pope D, Orton L, Bruce N. Using photovoice methods to explore older people's perceptions of respect and social inclusion in cities: opportunities, challenges and solutions. SSM - Popul Heal. 2016;2:732-44. https://doi.org/10.1016/j.ssmph.2016.09.004.

13. Doran P, Buffel T. Translating research into action. Manchester; 2017.

14. Scharlach $A E$, Lehning AJ. Ageing-friendly communities and social inclusion in the United States of America. Ageing Soc. 2013;33:110-36. https://doi. org/10.1017/S0144686X12000578.

15. Scharlach AE. Age-friendly cities: for whom? By whom? For what purpose? In: Moulaert T, Garon S, editors. Age-friendly cities communities int. comp: Springer; 2016. p. 305-29.
16. Ogg J, Renaut S. Social inclusion of elders in familiese. In: Scharf T, Keating NC, editors. From exclusion to Incl. old age. In: Policy; 2012

17. Officer A, Schneiders L, Wu D, Nash P, Thiyagarajan A. Valuing older people: time for a global campaign to combat ageism. Bull World Health Organ. 2016;94:710-710A. https://doi.org/10.2471/BLT.16.184960.

18. Nelson TD. Ageism: prejudice against our feared future self. J Soc Issues. 2005;61:207-21. https://doi.org/10.1111/j.1540-4560.2005.00402.x.

19. Angus J, Reeve P. Ageism: a threat to "aging well" in the 21 st century. J Appl Gerontol. 2006;25:137-52. https://doi.org/10.1177/0733464805285745.

20. Levy BR. Mind matters: cognitive and physical effects of aging self-stereotypes. Journals Gerontol Ser B. 2003;58:P203-11. https:/doi.org/10.1093/geronb/58.4.P203.

21. Levy BR, Slade MD, Murphy TE, Gill TM. Association between positive age stereotypes and recovery from disability in older persons. JAMA. 2012;308: 1972-3. https://doi.org/10.1001/jama.2012.14541.

22. Levy BR, Zonderman AB, Slade MD, Ferrucci L. Age stereotypes held earlier in life predict cardiovascular events in later life. Psychol Sci. 2009;20:296-8. https://doi.org/10.1111/j.1467-9280.2009.02298.x.

23. Cass N, Shove E, Urry J. Social exclusion, mobility and access. Sociol Rev. 2005:53:539-55. https://doi.org/10.1111/j.1467-954X.2005.00565.X.

24. Eriksson M, Bauer GF, Pelikan JM. The handbook of salutogenesis: Springer International; 2016.

25. Warburton J, Ng SH, Shardlow SM. Social inclusion in an ageing world: introduction to the special issue. Ageing Soc. 2012;33:1-15. https://doi.org/ 10.1017/S0144686X12000980.

26. Scharlach $A E$, Lehning AJ. Ageing-friendly communities and social inclusion in the United States of America. Ageing Soc. 2012;33:110-36. https://doi. org/10.1017/S0144686X12000578.

27. Daly M, Silver H. Social exclusion and social capital: a comparison and critique. Theory Soc. 2008;37:537-66. https://doi.org/10.1007/s11186-008-9062-4.

28. WHO. Why should cities become more age-friendly? 2015. https://extranet. who.int/agefriendlyworld/why-become-more-af/. Accessed 2 Oct 2017.

29. Petticrew M, Anderson L, Elder R, Grimshaw J, Hopkins D, Hahn R, et al. Complex interventions and their implications for systematic reviews: a pragmatic approach. J Clin Epidemiol. 2013;66:1209-14. https://doi.org/10. 1016/j.jclinepi.2013.06.004

30. Australian Public Service. Tackling wicked problems - a public policy perspective. Camberra; 2007.

31. Ogilvie D, Cummins S, Petticrew M. Assessing the evaluability of complex public health interventions: five questions for researchers, funders, and policymakers. The Milbank Quartelry. 2011;89:206-25.

32. Pawson R, Walshe K, Greenhalgh T. Realist synthesis: an introduction 2004. (ESRC Research Methods Programme RMP Me). London: ESRC Working Paper Series.

33. Koelen MA. Health promotion research: dilemmas and challenges. J Epidemiol Community Heal. 2001;55:257-62. https://doi.org/10.1136/jech.55.4.257.

34. Craig P, Dieppe P, Macintyre S, Michie S, Nazareth I, Petticrew M. Developing and evaluating complex interventions: the new Medical Research Council guidance. BMJ. 2008;337:a1655-5. https://doi.org/10.1136/bmj.a1655.

35. Lorenc T, Felix L, Petticrew M, Melendez-Torres GJ, Thomas J, Thomas S, et al. Meta-analysis, complexity, and heterogeneity: a qualitative interview study of researchers' methodological values and practices. Syst Rev. 2016;5: 192. https://doi.org/10.1186/s13643-016-0366-6.

36. Wong G. Is complexity just too complex? J Clin Epidemiol. 2013;66:1199201. https://doi.org/10.1016/j.jclinepi.2013.06.019.

37. Menec VH, Novek S, Veselyuk D, McArthur L. Learned from a Canadian province-wide, age-friendly initiative: the age-friendly Manitoba initiative. J Aging Soc Policy. 2013:37-41. https://doi.org/10.1080/08959420.2014.854606.

38. Buckner S, Pope D, Mattocks C, Lafortune L, Dherani M, Bruce N. Developing age-friendly cities: an evidence-based evaluation tool. J Popul Ageing. 2017; https://doi.org/10.1007/s12062-017-9206-2.

39. Orton L, Halliday E, Collins M, Egan M, Lewis S, Ponsford R, et al. Putting context centre stage: evidence from a systems evaluation of an area based empowerment initiative in England. Crit Public Health. 2016:1-13. https:// doi.org/10.1080/09581596.2016.1250868.

40. Centre for Reviews and Dissemination. Systematic reviews: CRD's guidance for undertaking reviews in health care. vol. 10. York: University of York; 2009.

41. Moher D, Liberati A. Preferred reporting items for systematic reviews and meta-analyses: the PRISMA statement. Ann Intern Med. 2009;339:332-6. https://doi.org/10.1136/bmj.b2535.

42. Ronzi S, Pope D, Orton L, Valtorta N, Bruce N. Clarifying the relationship between respect, social inclusion and health in older people: a systematic review of associations and intervention impacts. 
2014. https://www.crd.york.ac.uk/PROSPERO/display_record. php?RecordID=10107. Accessed 2 Oct 2017.

43. Anderson LM, Petticrew M, Rehfuess E, Armstrong R, Ueffing E, Baker P, et al. Using logic models to capture complexity in systematic reviews. Res Synth Methods. 2011;2:33-42. https://doi.org/10.1002/jrsm.32.

44. Anderson LM, Petticrew M, Chandler J, Grimshaw J, Tugwell P, O'Neill J, et al. Introducing a series of methodological articles on considering complexity in systematic reviews of interventions. J Clin Epidemiol. 2013;66: 1205-8. https://doi.org/10.1016/j.jclinepi.2013.07.005.

45. Squires JE, Valentine JC, Grimshaw JM. Systematic reviews of complex interventions: framing the review question. J Clin Epidemiol. 2013;66:121522. https://doi.org/10.1016/j.jclinepi.2013.05.013.

46. Petticrew M, Chalabi Z, Jones D. To RCT or not to RCT: deciding when "more evidence is needed" for public health policy and practice. Epidemiol Community Heal. 2012:391-6. https://doi.org/10.1136/jech.2010.116483.

47. Ogilvie D, Fayter D, Petticrew M, Sowden A, Thomas S, Whitehead M, et al. The harvest plot: a method for synthesising evidence about the differential effects of interventions. BMC Med Res Methodol. 2008;8:8. https://doi.org/ 10.1186/1471-2288-8-8.

48. Petticrew M. When are complex interventions "complex"? When are simple interventions "simple"? Eur J Pub Health. 2011;21:397-8. https://doi.org/10. 1093/eurpub/ckr084

49. Datta J, Petticrew M. Challenges to evaluating complex interventions: a content analysis of published papers. BMC Public Health. 2013;13:568. https://doi.org/10.1186/1471-2458-13-568.

50. Petticrew M, Rehfuess E, Noyes J, Higgins JPT, Mayhew A, Pantoja T, et al. Synthesizing evidence on complex interventions: how meta-analytical qualitative, and mixed-method approaches can contribute. J Clin Epidemiol. 2013;66:1230-43. https://doi.org/10.1016/j.jclinepi.2013.06.005.

51. Lui C-W, Everingham J-A, Warburton J, Cuthill M, Bartlett H. What makes a community age-friendly: a review of international literature. Australas $J$ Ageing. 2009;28:116-21. https://doi.org/10.1111/j.1741-6612.2009.00355.x

52. Steels $\mathrm{S}$. Key characteristics of age-friendly cities and communities: a review. Cities. 2015;47:45-52. https://doi.org/10.1016/j.cities.2015.02.004.

53. Buffel T, McGarry P, Phillipson C, De Donder L, Dury S, De Witte N, et al. Developing age-friendly cities: case studies from Brussels and Manchester and implications for policy and practice. J Aging Soc Policy. 2013:37-41. https://doi.org/10.1080/08959420.2014.855043.

54. Phillipson C, White S. Faheem Aftab. Old Moat: age-friendly neighbourhood report. Manchester; 2013.

55. Phillipson C. Developing age-friendly cities: policy challenges \& options. 2012.

56. Cattan M, Kime N, Bagnall AM. The use of telephone befriending in low level support for socially isolated older people —an evaluation. Heal Soc Care Community. 2011;19:198-206. https:/doi.org/10.1111/j.1365-2524.2010.00967.x.

57. Eppi Centre. EPPI-Reviewer 4 software for research synthesis user manual; 2015. p. 1-137. https://eppi.ioe.ac.uk/cms/Portals/35/Manuals/ER4.5. 0\%20user\%20manuala.pdf?ver=2015-10-12-122019-620. Accessed 2 Oct 2017

58. Katikireddi SV, Egan M, Petticrew M. How do systematic reviews incorporate risk of bias assessments into the synthesis of evidence? A methodological study. J Epidemiol Community Health. 2015;69:189-95. https://doi.org/10. 1136/jech-2014-204711.

59. Atkins C, Sampson J. Critical appraisal guidelines for single case study research. Ecis. 2002:100-9.

60. Pope D. Liverpool quality assessment tools 2014. Oral Communication.

61. Rehfuess EA, Puzzolo E, Stanistreet D, Pope D, Bruce NG. Enablers and barriers to large-scale uptake of improved solid fuel stoves: a systematic review. Environ Health Perspect. 2014;122:120-30. https://doi.org/10.1289/ehp.1306639.

62. Harden A, Brunton G, Fletcher A, Oakley A. Teenage pregnancy and social disadvantage: systematic review integrating controlled trials and qualitative studies. BMJ. 2009:339:b4254. https://doi.org/10.1136/bmj.b4254.

63. Mays N, Pope C. Qualitative research in health care: assessing quality in qualitative research. BMJ. 2000;320 https://doi.org/10.1111/j.1743-6109.2010.02151.x.

64. Popay J, Roberts H, Sowden A, Petticrew M, Arai L, Rodgers M, et al. Guidance on the conduct of narrative synthesis in systematic reviews - a product from the ESRC methods programme. 2006

65. Ellis SW. The Beth Johnson Foundation generations in action: final evaluation report (2004). 2004.

66. Cohen $\mathrm{G}$, Perlstein $\mathrm{S}$. The impact of professionally conducted cultural programs on the physical health, mental health, and social functioning of older adults. Gerontologist. 2006;46:726-34.
67. Slegers $K$, van Boxtel M, Jolles J. Effects of computer training and internet usage on the well-being and quality of life of older adults: a randomized, controlled study. 879 Gerontol B Psychol Sci Soc Sci. 2008; 63:176-84.

68. Chung JCC. An intergenerational reminiscence programme for older adults with early dementia and youth volunteers: values and challenges. Scand J Caring Sci. 2009;23:259-64. https://doi.org/10.1111/j.1471-6712.2008.00615.x.

69. Davidson JW, Fedele J. Investigating group singing activity with people with dementia and their caregivers: problems and positive prospects. Music Sci. 2011;15:402-22. https://doi.org/10.1177/1029864911410954.

70. Houston S, McGill A. English National Ballet Dance for Parkinson's. 2015. https://www.ballet.org.uk/wp-content/uploads/2017/03/English-NationalBallet-Dance-for-Parkinsons-research-report.pdf. Accessed 2 Oct 2017

71. Hackney ME, Kantorovich S, Earhart GM. A study on the effects of argentine tango as a form of partnered dance for those with Parkinson disease and the healthy elderly. Am J Danc Ther. 2007;29:109-27. https://doi.org/10. 1007/s10465-007-9039-2.

72. Houston S, McGill A. English National Ballet Dance for Parkinson's: An investigative study. 2011. https://hafraah.files.wordpress.com/2015/02/dancefor-parkinsons-report-2011.pdf. Accessed 2 Oct 2017.

73. Ruffing-Rahal MA. Evaluation of group health promotion with communitydwelling older women. Public Health Nurs. 1994;11:38-48.

74. Vogelpoel N, Jarrold K. Social prescription and the role of participatory arts programmes for older people with sensory impairments. J Integr Care. 2014; 22:39-50. https://doi.org/10.1108/JICA-01-2014-0002.

75. Gaggioli A, Morganti L, Bonfiglio S, Scaratti C, Cipresso P, Serino S, et al. Intergenerational group reminiscence: a potentially effective intervention to enhance elderly psychosocial wellbeing and to improve children's perception of aging. Educ Gerontol. 2014;40:486-98. https://doi.org/10.1080/ 03601277.2013.844042

76. Fried LP, Carlson MC, Freedman M, Frick KD, Glass TA, Hill J, et al. A social model for health promotion for an aging population: initial evidence on the Experience Corps model. J Urban Heal. 2004;81:64-78. https://doi.org/10.1093/jurban/jth094.

77. Dickens AP, Richards SH, Hawton A, Taylor RS, Greaves CJ, Green C, et al. An evaluation of the effectiveness of a community mentoring service for socially isolated older people: a controlled trial. BMC Public Health. 2011;11: 218. https://doi.org/10.1186/1471-2458-11-218.

78. Woodward AT, Freddolino PP, Wishart DJ, Bakk L, Kobayashi R, Tupper C, et al. Outcomes from a peer tutor model for teaching technology to older adults. Ageing Soc. 2012:1-24. https://doi.org/10.1017/S0144686X12000530.

79. Kocken PL, Voorham a JJ. Effects of a peer-led senior health education program. Patient Educ Couns. 1998;34:15-23. https://doi.org/10.1016/S07383991(98)00042-1.

80. Schlag PA. Older adults' computer use: a case study of participants' involvement with a SeniorNet program. Int J Disabil Hum Dev. 2011;10:13944. https://doi.org/10.1515/JJDHD.2011.026.

81. De Souza EM, Grundy E. Intergenerational interaction, social capital and health: results from a randomised controlled trial in Brazil. Soc Sci Med. 2007;65:1397-409. https://doi.org/10.1016/.jsocscimed.2007.05.022.

82. Hong SI, Morrow-Howell N. Health outcomes of Experience Corps ${ }^{\oplus}$ : a highcommitment volunteer program. Soc Sci Med. 2010;71:414-20. https://doi. org/10.1016/j.socscimed.2010.04.009.

83. Fujiwara $Y$, Sakuma $N$, Ohba H, Nishi M, Lee $S$, Watanabe $N$, et al. REPRINTS: effects of an intergenerational health promotion program for older adults in Japan. J Intergener Relatsh. 2009;7:17-39. https://doi.org/10.1080/ 15350770802628901

84. Murayama Y, Ohba H, Yasunaga M, Nonaka K, Takeuchi R, Nishi M, et al. The effect of intergenerational programs on the mental health of elderly adults. Aging Ment Health. 2014;19:306-14. https://doi.org/10. 1080/13607863.2014.933309.

85. Ellis SW. Changing the lives of children and older people: intergenerational mentoring in secondary schools. 2003. Beth Johnson Foundation and Manchester: Metropolitan University.

86. De Souza EM. Intergenerational interaction in health promotion: a qualitative study in Brazil. Rev Saude Publica. 2003;37:463-9.

87. Hernandez CR, Gonzalez MZ. Effects of intergenerational interaction on aging. Educ Gerontol. 2008;34:292-305. https://doi.org/10.1080/ 03601270701883908

88. Gonyea JG, Burnes K. Aging well at home: evaluation of a neighborhoodbased pilot project to "put connection back into community". J Hous Elderly. 2013;27:333-47. https://doi.org/10.1080/02763893.2013.813425. 
89. Weintraub PC, Killian TS. Intergenerational programming: older persons' perceptions of its impact. J Appl Gerontol. 2007;26:370-84. https://doi.org/ 10.1177/0733464807302671.

90. Buijs R, Ross-Kerr J, Cousins SO, Wilson D. Promoting participation: evaluation of a health promotion program for low income seniors. J Community Health Nurs. 2003;20:93-107. https://doi.org/10.1207/ S15327655JCHN200203.

91. Coulton S, Clift S, Skingley A, Rodriguez J. Effectiveness and costeffectiveness of community singing on mental health-related quality of life of older people: randomised controlled trial. Br J Psychiatry. 2015:250-5. https://doi.org/10.1192/bjp.bp.113.129908.

92. Clift S, Morrison I. Group singing fosters mental health and wellbeing: findings from the East Kent "singing for health" network project. Ment Heal Soc Incl. 2011;15:88-97. https://doi.org/10.1108/20428301111140930.

93. Creech A, Hallam S, Varvarigou M, McQueen H, Gaunt H. Active music making: a route to enhanced subjective well-being among older people. Perspect Public Health. 2013;133:36-43. https://doi.org/10.1177/ 1757913912466950.

94. Davidson JW, McNamara B, Rosenwax L, Lange A, Jenkins S, Lewin G. Evaluating the potential of group singing to enhance the well-being of older people. Australas J Ageing. 2014;33:99-104. https://doi.org/10.1111/j. 1741-6612.2012.00645.x.

95. Woodward A, Freddolino P, Blaschke-Thompson C, Wishart D, Bakk L, Kobayashi R, et al. Technology and aging project: training outcomes and efficacy from a randomized field trial. Ageing Int. 2011;36:46-65. https://doi. org/10.1007/s12126-010-9074-z.

96. Phinney A, Moody EM, Small JA. The effect of a community-engaged arts program on older adults' well-being. Can J Aging. 2014;33:336-45. https:// doi.org/10.1017/S071498081400018X.

97. Camic PM, Tischler V, Pearman $\mathrm{CH}$. Viewing and making art together: a multi-session art-gallery-based intervention for people with dementia and their carers. Aging Ment Health. 2014;18:161-8. https://doi.org/10.1080/ 13607863.2013.818101.

98. Yuen HK, Mueller K, Mayor E, Azuero A. Impact of participation in a theatre programme on quality of life among older adults with chronic conditions: a pilot study. Occup Ther Int. 2011;18:201-8. https://doi.org/10.1002/oti.327.

99. Saito T, Kai I, Takizawa A. Effects of a program to prevent social isolation on loneliness, depression, and subjective well-being of older adults: a randomized trial among older migrants in Japan. Arch Gerontol Geriatr. 2012;55:539-47. https://doi.org/10.1016/j.archger.2012.04.002.

100. Greaves CJ. Effects of creative and social activity on the health and wellbeing of socially isolated older people: outcomes from a multi-method observational study. J R Soc Promot Heal. 2006;126:134-42. https://doi.org/ $10.1177 / 1466424006064303$.

101. VarVarigou M, CreeCh A, HallaM S, CQueen H. Benefits experienced by older people in group music-making activities. J Appl Arts Heal. 2012; https://doi. org/10.1386/jaah.3.2.183.

102. Skingley $A$, Bungay $H$. The Silver Song Club Project: singing to promote the health of older people. Br J Community Nurs. 2010;15:135-40. https://doi. org/10.12968/bjen.2010.15.3.46902.

103. Mendis KP. The effects of participation in an intergenerational programme on the psychological wellbeing of the elderly: Fordham University; 1993. https://doi.org/10.16953/deusbed.74839.

104. Newman S, Karip E, Faux RB. Everyday memory function of older adults: the impact of intergenerational school volunteer programs. J Educ Gerontol. 1995;21

105. Varvarigou M, Creech A, Hallam S, McQueen H. Bringing different generations together in music-making: an intergenerational music project in East London. Int J Community Music. 2011;4:207-20. https://doi.org/10. 1386/ijcm.4.3.207_1.

106. Gonyea JG, Burnes K. Aging well at home: evaluation of a neighborhoodbased pilot project to "put connection back into community". J Hous Elderly 2013;27:333-347 15p. doi:https://doi.org/10.1080/02763893.2013.813425.

107. Coulton S, Clift S, Skingley A, Rodriguez J. Effectiveness and costeffectiveness of community singing on mental health-related quality of life of older people: randomised controlled trial. Br J Psychiatry. 2015; https:// doi.org/10.1192/bjp.bp.113.129908.

108. Skingley A, Clift SM, Coulton SP, Rodriguez J. The effectiveness and costeffectiveness of a participative community singing programme as a health promotion initiative for older people: protocol for a randomised controlled trial. BMC Public Health. 2011;11:142. https://doi.org/10.1186/ 1471-2458-11-142
109. Camic PM, Williams CM, Meeten F. Does a "singing together group" improve the quality of life of people with a dementia and their carers? A pilot evaluation study. Dementia. 2013;12:157-76. https://doi.org/10.1177/ 1471301211422761

110. Yen $I H$, Michael $Y L$, Perdue L. Neighborhood environment in studies of health of older adults: a systematic review. Am J Prev Med. 2009;37:455-63. https://doi.org/10.1016/j.amepre.2009.06.022.

111. Dickens A, Richards S, Greaves C, Campbell J. Interventions targeting social isolation in older people: a systematic review. BMC Public Health. 2011;11: 647. https://doi.org/10.1186/1471-2458-11-647.

112. Cattan M. Preventing social isolation and loneliness among older people: a systematic review of health promotion interventions. Ageing Soc. 2005;25: 41-67. https://doi.org/10.1017/S0144686X04002594.

113. Medical Advisory Secretariat. Dwelling seniors: an evidence based analysis. Ont Health Technol Assess Ser. 2008;8

114. Findlay R. Interventions to reduce social isolation amongst older people: where is the evidence? Ageing Soc. 2003;23 https://doi.org/10.1017/ S0144686X03001296.

115. Hagan R, Manktelow R, Taylor BJ, Mallett J. Reducing loneliness amongst older people: a systematic search and narrative review. Aging Ment Health. 2014;18:683-93. https://doi.org/10.1080/13607863.2013.875122.

116. Choi M, Kong S, Jung D. Computer and internet interventions for loneliness and depression in older adults: a meta-analysis. Healthc Inform Res. 2012;18: 191-8. https://doi.org/10.4258/hir.2012.18.3.191.

117. Milligan C, Neary D, Payne S, Hanratty B, Irwin P, Dowrick C. Older men and social activity: a scoping review of Men's Sheds and other gendered interventions. Ageing Soc. 2015:1-29. https://doi.org/10.1017/ S0144686X14001524

118. Lafortune L, Kelly S, Olanrewaju O, Cowan A. Changing risk behaviours and promoting cognitive health in older adults an evidence-based resource for local authorities and commissioners. London: Public Health England; 2016. https://www.gov.uk/ government/publications/changing-risk-behaviours-and-promotingcognitive-health-in-older-adults. Accessed 2 Oct 2017

119. Heaven B, Brown LJE, White M, Errington L, Mathers JC, Moffatt S. Supporting well-being in retirement through meaningful social roles: systematic review of intervention studies. Milbank Q. 2013;91:222-87. https://doi.org/10.1111/milq.12013.

120. Centre for Reviews and Dissemination. Interventions for loneliness and social isolation. 2014. https://www.york.ac.uk/media/crd/ Loneliness\%20and\%20social\%20isolation.pdf. Accessed 2 Oct 2017.

121. Clift S, Hancox G, Staricoff R, Christine W. Siging and health: a systematic mapping and review of non-clinical research. Sydney; 2008.

122. Keogh JWL, Kilding A, Pidgeon P, Ashley L, Gillis D. Physical benefits of dancing for healthy older adults: a review. J Aging Phys Act. 2009;17:479-500.

123. Lötzke D, Ostermann T, Büssing A. Argentine tango in Parkinson disease-a systematic review and meta-analysis. BMC Neurol. 2015;15:226. https://doi. org/10.1186/s12883-015-0484-0.

124. Egger M, Zellweger-Zahner T. Language bias in randomised controlled trials published in English and German. Lancet. 1997;350:326-9.

125. Pope DP, Mishra V, Thompson L, Siddiqui AR, Rehfuess EA, Weber M, et al. Risk of low birth weight and stillbirth associated with indoor air pollution from solid fuel use in developing countries. Epidemiol Rev. 2010;32:70-81. https://doi.org/10.1093/epirev/mxq005.

126. Puzzolo E, Pope D, Stanistreet D, Rehfuess EA, Bruce NG. Clean fuels for resource-poor settings: a systematic review of barriers and enablers to adoption and sustained use. Environ Res. 2016;146:218-34. https://doi.org/ 10.1016/j.envres.2016.01.002.

127. Voss PH, Rehfuess EA. Quality appraisal in systematic reviews of public health interventions: an empirical study on the impact of choice of tool on meta-analysis. J Epidemiol Community Health. 2013;67:98-104. https://doi. org/10.1136/jech-2011-200940.

128. Petticrew M. Time to rethink the systematic review catechism? Moving from "what works" to "what happens". Syst Rev. 2015;4:36. https://doi.org/10. 1186/s13643-015-0027-1

129. Whitehead M, Dahlgren G. Concepts and principles for tackling social inequities in health: levelling up. Part 1. Copenhagen: World Health Organization; 2007.

130. Marmot M. Fair society, healthy lives. vol. 126 Suppl. 2010. doi:https://doi. org/10.1016/j.puhe.2012.05.014. http://www.enothe.eu/cop/docs/concepts_ and_principles.pdf. Accessed 2 Jun 2017. 
131. McCambridge J, Witton J, Elbourne DR. Systematic review of the Hawthorne effect: new concepts are needed to study research participation effects. J Clin Epidemiol. 2014;67:267-77. https://doi.org/10.1016/j.jlinepi.2013.08.015.

132. Rebok GW, Carlson MC, Barron JS, Frick KD, Mcgill S, Parisi JM, et al.

Experience Corps ${ }^{\ominus}$ a civic engagement-based public health intervention in the public schools; 2011. https://doi.org/10.1007/978-1-4419-0636-6.

133. Clift S, Skingley A, Coulton S, Rodriguez J. A controlled evaluation of the health benefits of a participative community singing programme for older people (Silver Song Clubs). 2012. https://www.canterbury.ac.uk/health-andwellbeing/sidney-de-haan-research-centre/documents/community-singingprogramme-for-older-people.pdf. Accessed 2 Oct 2017.

134. Hallam S, Creech A, Varvarigou M, McQueen H. Perceived benefits of active engagement with making music in community settings. Int J Community Music. 2012;5:155-74. https://doi.org/10.1386/ijcm.5.2.155.

135. Slegers K, van Boxtel MP. Actual use of computers and the internet by older adults: potential benefits and risks. In: Slegers $\mathrm{K}$, van Boxtel MP, editors. Engag. older adults with Mod. Technol: Internet use Inf. access needs: IGI Global; 2013. p. 161-90. https://doi.org/10.4018/978-1-4666-1966-1.ch009.

136. Weintraub APC, Killian TS. Perceptions of the impact of intergenerational programming on the physical well-being of participants in adult day services. J Intergener Relatsh. 2009;7:355-70. https://doi.org/10.1080/ 15350770903285312

\section{Submit your next manuscript to BioMed Central and we will help you at every step:}

- We accept pre-submission inquiries

- Our selector tool helps you to find the most relevant journal

- We provide round the clock customer support

- Convenient online submission

- Thorough peer review

- Inclusion in PubMed and all major indexing services

- Maximum visibility for your research

Submit your manuscript at www.biomedcentral.com/submit

C) Biomed Central 